Pacific

Journal of

Mathematics

STABILITY OF THE CONJUGATE INDEX, DEGENERATE CONJUGATE POINTS AND THE MASLOV INDEX IN SEMI-RIEMANNIAN GEOMETRY

Francesco Mercuri, Paolo Piccione, and Daniel V. Tausk 


\title{
STABILITY OF THE CONJUGATE INDEX, DEGENERATE CONJUGATE POINTS AND THE MASLOV INDEX IN SEMI-RIEMANNIAN GEOMETRY
}

\author{
Francesco Mercuri, Paolo Piccione, and Daniel V. Tausk
}

We investigate the problem of the stability of the number of conjugate or focal points (counted with multiplicity) along a semi-Riemannian geodesic $\gamma$. For a Riemannian or a nonspacelike Lorentzian geodesic, such number is equal to the intersection number (Maslov index) of a continuous curve with a subvariety of codimension one of the Lagrangian Grassmannian of a symplectic space. In the general semi-Riemannian case, under a certain nondegeneracy assumption on the conjugate points, this number is equal to an algebraic count of their multiplicities. In this paper we reprove some results that were incorrectly stated by Helfer in 1994, where the occurrence of degeneracies was overlooked; in particular, a counterexample to one of Helfer's results, which is essential for the theory, is given. In the last part of the paper we discuss a general technique for the construction of examples and counterexamples in the index theory for semi-Riemannian geodesics, in which some new phenomena appear.

\section{Introduction.}

The original motivation for writing this paper was given by the following problem. Given a sequence $\gamma_{j}$ of geodesics in a semi-Riemannian manifold $(\mathcal{M}, \mathfrak{g})$ converging to a geodesic $\gamma_{\infty}$, what can be said about the convergence of the conjugate index of $\gamma_{j}$ to that of $\gamma_{\infty}$ ? The question arose in the context of Lorentzian geometry, where the problem originated in an attempt to develop a Morse theory for lightlike geodesics as a limit of the theory for timelike geodesics (see [11]). Recall that the conjugate index of a geodesic $\gamma:[a, b] \rightarrow \mathcal{M}$ is the number of points that are conjugate to $\gamma(a)$ along $\gamma$, counted with multiplicity. Conjugate points along a geodesic correspond to the zeroes of (nontrivial) Jacobi fields along $\gamma$, which are vector fields in the kernel of the second variation of the action functional $z \mapsto \int_{a}^{b} \mathfrak{g}\left(z^{\prime}, z^{\prime}\right) \mathrm{d} t$, called the index form $I_{\gamma}$. The celebrated Morse Index Theorem (see for instance $[\mathbf{2}, \mathbf{3}, \mathbf{6}, \mathbf{7}, \mathbf{9}, \mathbf{1 6}, \mathbf{1 7}]$ for versions of this theorem in different contexts) states that the conjugate index of a Riemannian or nonspacelike Lorentzian 
geodesic $\gamma$ is equal to the index of $I_{\gamma}$, provided that the final point $\gamma(b)$ is not considered in the count of conjugate points. It is not too hard to prove that the convergence of a sequence of geodesics to a geodesic implies the norm convergence of the correspondent index forms, seen as bilinear forms on the Hilbert space of $H^{1}$ variational vector fields. Hence, the problem of convergence of the conjugate index can be reformulated in terms of convergence of the index on a sequence of self-adjoint operators converging in the operator norm. It follows from the spectral theorem that the index of a self-adjoint operator is indeed stable by small perturbations in the norm topology, provided that the operator be invertible. If $(\mathcal{M}, \mathfrak{g})$ is Riemannian, i.e., $\mathfrak{g}$ is positive definite, then the self-adjoint operator associated to the index form along the limit geodesic $\gamma_{\infty}$ is indeed invertible, provided that the point $\gamma_{\infty}(b)$ is not conjugate to $\gamma_{\infty}(a)$ along $\gamma_{\infty}$; thus, the Morse index theorem and the stability of the index of an invertible self-adjoint operator on a Hilbert space imply that the conjugate index of $\gamma_{j}$ is eventually constant, and equal to the conjugate index of $\gamma_{\infty}$. The same conclusion holds for timelike Lorentzian geodesics, provided that the limit be also timelike. When one considers the case of lightlike geodesics, though, the situation is complicated by the fact that the index form always has a nontrivial kernel, even when the final endpoint is nonconjugate to the initial one. Thus, using functional-analytical arguments one only proves the semi-continuity of the index for Lorentzian nonspacelike geodesics.

A different technique to study the stability of the index is suggested by an analogy with the classical Sturm problem in the theory of ordinary differential equations. The Sturm oscillation theorem ([5, Chapter 8$]$ ) gives the equality between the index of a certain symmetric bilinear form $B$ and the number of zeroes of a solution of the Sturm equation. Its proof is obtained by showing that the two quantities involved in the thesis can be obtained as the winding number of two homotopic closed curves in the real projective line. As a side effect of this theory, one obtains immediately that, since the winding number is stable by small $C^{0}$-perturbations, the index of $B$ is stable by small perturbations. So, the stability of the index for a Sturm system is proven by relating the index form $B$ to some homotopical invariants of the problem. In this paper we exploit this idea to obtain results on the stability of the conjugate index for semi-Riemannian geodesics. More precisely, we investigate the notion of Maslov index for a Jacobi type system of ordinary differential equations, which is obtained as the intersection number between a curve $\ell$ determined by the flow of the system and a subvariety of codimension one of the Lagrangian Grassmannian of a symplectic space. The zeroes of the solutions of the Jacobi type system correspond to the intersections of the curve $\ell$ with the subvariety; moreover, the Maslov index of the system arising from the Jacobi equation along a Riemannian or a nonspacelike 
Lorentzian geodesic is proven to be equal to the conjugate index of the geodesic. In the general semi-Riemannian case, under a suitable nondegeneracy assumption on the conjugate points, the Maslov index corresponding to a geodesic is proven to be equal to an algebraic count of the conjugate points, which we also call the conjugate index of the semi-Riemannian geodesic.

The idea of studying conjugate points in differential equations via intersection theory is not new. In [4] and [8] it is developed an approach to the index problem through topological methods. For instance, in [8], it is employed a similar technique to prove a generalization of Sturm's Theorems in the case of an arbitrary self-adjoint system of even order subject to arbitrary self-adjoint boundary conditions. In [14], the author carries out a similar analysis for Morse-Sturm-Liouville systems which are obtained from the Jacobi equation along a semi-Riemannian geodesic. The intersection theory employed is based on the notion of Maslov index of a curve which is defined in [14] using homology/cohomology duality on the Lagrangian Grassmannian. This approach does not seem to deal properly with the lack of orientability of the Lagrangian Grassmannian (see [10]). Furthermore, several results of Helfer's paper are incorrectly stated, due mainly to the lack of an essential assumption of nondegeneracy for the restrictions of certain symmetric bilinear forms. More precisely, in [14, Proposition 5.1 (b)] it is claimed the equality between the Maslov index and the sum of the signatures of the conjugate points along a semi-Riemannian geodesic; we give a counterexample to such equality in Subsection 5.4. For these reasons, we have opted to provide an alternative, self-contained, presentation of the definition and some basic properties of the Maslov index. It is important to observe that the kind of degeneracy discussed above, which do not occur in Riemannian or nonspacelike Lorentzian geodesics, is responsible for many of the new phenomena which arise in the general semi-Riemannian case, like for instance, the possibility of accumulation of the conjugate points along a geodesic. Curiously enough, also in the book by O'Neill [15, Exercise 8, page 299], the nondegeneracy assumption is missing, and the author claims incorrectly that the set of focal points along a geodesic is discrete.

The paper is organized as follows. In Section 2 we introduce the basic notation and we discuss some preliminary facts. Section 3 is devoted to the intersection theory used for the definition and the properties of the Maslov index. We remark that the geometry of the Lagrangian Grassmannian has already been studied by several authors (see for instance $[\mathbf{1}, \mathbf{1 3}, \mathbf{1 8}]$ ). The techniques of computation of the Maslov index in terms of change of signatures of symmetric bilinear forms presented in Section 3 are very practical for our purposes of relating the Maslov index and the conjugate index of a geodesic (see Theorem 4.3 and the following corollary). 
In Section 4 we apply the results of the previous sections to the problem of the stability of the conjugate and focal indexes in semi-Riemannian geometry. A special attention is given to the case of the approximation of a lightlike geodesic by timelike geodesics in a Lorentzian manifold. If $\gamma_{\infty}$ is a lightlike geodesic in a Lorentzian manifold whose endpoints are not conjugate and $\gamma_{j}$ is a sequence of timelike geodesics converging to $\gamma_{\infty}$, then the conjugate index of $\gamma_{j}$ is eventually constant and equal to the conjugate index of $\gamma_{\infty}$. We also study the problem of stability in the case of geodesics $\gamma_{j}$ starting orthogonally to submanifolds $\mathcal{P}_{j}$, assuming a suitable notion of convergence for the initial data. It is a curious fact that, even in the Riemannian case, stability does not hold under the most general natural notion of convergence of the initial data (see Example 4.9).

In Section 5, we study the problem of determining which curves of Lagrangians are associated to solutions of Jacobi systems. We give some necessary and sufficient conditions for this occurrence, and we use these conditions to find an example in which the equality between the Maslov index and the conjugate index of a Lorentzian spacelike geodesic $\gamma$ fails, due to the degeneracy of the metric on the space $\mathfrak{J}[t]$ consisting of the evaluations at $t$ of the Jacobi fields along $\gamma$ that vanish at the initial instant.

\section{Preliminaries.}

Given vector spaces $V, W$, we denote by $\operatorname{Lin}(V, W)$ the space of linear maps from $V$ to $W$; all vector spaces will be assumed to be real and finitedimensional. We also denote by $\operatorname{Bil}(V, W)$ the space of bilinear forms $B$ : $V \times W \rightarrow \mathbb{R}$; when $V=W$ we write simply $\operatorname{Lin}(V)$ and $\operatorname{Bil}(V)$. We will always implicitly identify a bilinear map $B: V \times W \rightarrow \mathbb{R}$ with the linear map from $V$ to the dual space $W^{*}$ given by $v \mapsto B(v, \cdot)$. The space of symmetric bilinear forms $B: V \times V \rightarrow \mathbb{R}$ will be denoted by $\operatorname{Bil}_{\text {sym }}(V)$; observe that $B \in \operatorname{Bil}(V) \cong \operatorname{Lin}\left(V, V^{*}\right)$ is symmetric if and only if the transpose linear map $B^{*}: V^{* *} \cong V \rightarrow V^{*}$ is equal to $B$. If $S$ is a subspace of $V$ then we denote by $S^{\mathrm{o}} \subset V^{*}$ the annihilator of $S$ in $V$. Whenever $B \in \operatorname{Bil}_{\text {sym }}(V)$ is fixed by the context, we denote by $S^{\perp}=B^{-1}\left(S^{\circ}\right) \subset V$ the orthogonal complement of $S$ with respect to $B$. The index of a symmetric bilinear form $B \in \operatorname{Bil}_{\text {sym }}(V)$, denoted by $n_{-}(B)$, is defined as the maximum of the dimensions of the subspaces of $V$ on which $B$ is negative definite; the coindex of $B$, denoted by $n_{+}(B)$, is defined by $n_{+}(B)=n_{-}(-B)$ and the signature of $B$ is defined by $\operatorname{sgn}(B)=n_{+}(B)-n_{-}(B)$. The kernel of $B$, denoted by $\operatorname{Ker}(B)$, is the set $V^{\perp}$ of vectors $v \in V$ such that $B(v, w)=0$ for all $w \in V$; when $\operatorname{Ker}(B)=\{0\}$ we say that $B$ is nondegenerate.

2.1. The geometrical problem. Let $(\mathcal{M}, \mathfrak{g})$ be a semi-Riemannian manifold, i.e., $\mathcal{M}$ is a finite dimensional real differentiable manifold and $\mathfrak{g}$ is a smooth nondegenerate metric tensor on $\mathcal{M}$. We set $n=\operatorname{dim}(\mathcal{M})$. A nonzero 
vector $v \in T \mathcal{M}$ is called spacelike, timelike or lightlike according to $\mathfrak{g}(v, v)$ being positive, negative or null. The semi-Riemannian manifold $(\mathcal{M}, \mathfrak{g})$ is said to be Lorentzian if the index of $\mathfrak{g}$ at every point of $\mathcal{M}$ is equal to 1 . Let $\nabla$ denote the Levi-Civita connection of $\mathfrak{g}$ and let $\mathcal{R}$ denote the curvature tensor of $\nabla$ chosen with sign convention $\mathcal{R}(X, Y)=\nabla_{X} \nabla_{Y}-\nabla_{Y} \nabla_{X}-\nabla_{[X, Y]}$. If $V:[a, b] \rightarrow T \mathcal{M}$ is a vector field along a given curve $\gamma:[a, b] \rightarrow \mathcal{M}$, we will write $V^{\prime}$ for the covariant derivative of $V$ along $\gamma$. Let $\mathcal{P}$ be a smooth submanifold of $\mathcal{M}, p \in P$ and $\mathfrak{n} \in T_{p} \mathcal{P}^{\perp}$; by $\perp$ we mean the orthogonal complement with respect to $\mathfrak{g}$. The second fundamental form of $\mathcal{P}$ at $p$ in the normal direction $\mathfrak{n}$ is the symmetric bilinear form $\mathcal{S}_{\mathfrak{n}} \in \operatorname{Bil}_{\text {sym }}\left(T_{p} \mathcal{P}\right)$ given by $\mathcal{S}_{\mathfrak{n}}\left(v_{1}, v_{2}\right)=\mathfrak{g}\left(\nabla_{v_{1}} V_{2}, \mathfrak{n}\right)$, where $V_{2}$ is any extension of $v_{2}$ to a vector field on $\mathcal{P}$. If $\mathcal{P}$ is nondegenerate at $p$, i.e., if the restriction of $\mathfrak{g}$ to $T_{p} \mathcal{P}$ is nondegenerate, then there exists a unique linear endomorphism $A_{\mathfrak{n}}$ of $T_{p} \mathcal{P}$ such that $\mathcal{S}_{\mathfrak{n}}\left(v_{1}, v_{2}\right)=\mathfrak{g}\left(A_{\mathfrak{n}}\left(v_{1}\right), v_{2}\right)$ for all $v_{1}, v_{2} \in T_{p} \mathcal{P}$. Let $\gamma:[a, b] \rightarrow \mathcal{M}$ be a geodesic orthogonal to $\mathcal{P}$ at the initial point, i.e., $\gamma(a) \in \mathcal{P}$ and $\gamma^{\prime}(a) \in T_{\gamma(a)} \mathcal{P}^{\perp}$. We say that $\gamma$ is spacelike, timelike or lightlike according to $\gamma^{\prime}(t)$ being spacelike, timelike or lightlike for all $t$. Let's assume that $\mathcal{P}$ is nondegenerate at $\gamma(a)$; we will say that the family of objects $(\mathcal{M}, \mathfrak{g}, \gamma, \mathcal{P})$ is an admissible quadruple for the geometrical problem.

A Jacobi field along $\gamma$ is a smooth vector field $\mathcal{J}$ along $\gamma$ that satisfies the Jacobi equation:

$$
\mathcal{J}^{\prime \prime}(t)=\mathcal{R}\left(\gamma^{\prime}(t), \mathcal{J}(t)\right) \gamma^{\prime}(t)
$$

We say that $\mathcal{J}$ is a $\mathcal{P}$-Jacobi field if, in addition, $\mathcal{J}(a)$ satisfies:

$$
\mathcal{J}(a) \in T_{\gamma(a)} \mathcal{P} \quad \text { and } \quad \mathcal{J}^{\prime}(a)+A_{\gamma^{\prime}(a)}(\mathcal{J}(a)) \in T_{\gamma^{\prime}(a)} \mathcal{P}^{\perp}
$$

If $\mathcal{P}$ is a single point of $\mathcal{M}$, then (2.2) reduces to $\mathcal{J}(a)=0$. Geometrically, Equation (2.1) means that $\mathcal{J}$ is the variational vector field corresponding to a variation of $\gamma$ by geodesics; condition (2.2) says that these geodesics are orthogonal to $\mathcal{P}$ at their initial points. We set:

$$
\mathfrak{J}=\{\mathcal{J}: \mathcal{J} \text { is a } \mathcal{P} \text {-Jacobi field along } \gamma\}, \quad \mathfrak{J}[t]=\{\mathcal{J}(t): \mathcal{J} \in \mathfrak{J}\} .
$$

Observe that $\mathfrak{J}$ is an $n$-dimensional subspace of the space of all vector fields along $\gamma$ because the pairs $\left(\mathcal{J}(a), \mathcal{J}^{\prime}(a)\right) \in T_{\gamma(a)} \mathcal{M} \oplus T_{\gamma(a)} \mathcal{M}$ satisfying (2.2) form an $n$-dimensional subspace. A point $\gamma(t), t \in] a, b]$ is said to be $\mathcal{P}$-focal along $\gamma$ if there exists a nonzero $\mathcal{J} \in \mathfrak{J}$ with $\mathcal{J}(t)=0$; the multiplicity of $\gamma(t)$, denoted by mul $(t)$, is defined to be the dimension of the space of those $\mathcal{J} \in \mathfrak{J}$ with $\mathcal{J}(t)=0$. If $\gamma(t)$ is not $\mathcal{P}$-focal we set $\operatorname{mul}(t)=0$. Since $\operatorname{dim}(\mathfrak{J})=\operatorname{dim}\left(T_{\gamma(t)} \mathcal{M}\right)=n$, then:

$$
\operatorname{mul}(t)=\operatorname{codim}(\mathfrak{J}[t])=\operatorname{dim}\left(\mathfrak{J}[t]^{\perp}\right) .
$$

When $\mathcal{P}$ reduces to a point then the $\mathcal{P}$-focal points are also called conjugate points. 
Definition 2.1. The signature of a $\mathcal{P}$-focal point $\gamma(t)$, denoted by $\operatorname{sgn}(t)$, is defined to be the signature of the restriction of the metric $\mathfrak{g}$ to the space $\mathfrak{J}[t]^{\perp}$; if $\gamma(t)$ is not a $\mathcal{P}$-focal point, we set $\operatorname{sgn}(t)=0$. A $\mathcal{P}$-focal point $\gamma(t)$ is called nondegenerate if the restriction of $\mathfrak{g}$ to $\mathfrak{J}[t]$ (or, equivalently, the restriction of $\mathfrak{g}$ to $\mathfrak{J}[t]^{\perp}$ ) is nondegenerate. The focal index of the geodesic $\gamma$ relative to $\mathcal{P}$ is defined by $\mathrm{i}_{\text {foc }}(\gamma, \mathcal{P})=\sum_{t \in] a, b]} \operatorname{sgn}(t)$, provided that the number of $\mathcal{P}$-focal points along $\gamma$ is finite (see Proposition 2.2 and Remark 2.3). When the submanifold $\mathcal{P}$ reduces to a point, the focal index is also called the conjugate index.

If $\mathfrak{g}$ is Riemannian or if $\mathfrak{g}$ is Lorentzian and $\gamma$ is nonspacelike then $\mathfrak{g}$ is positive definite on $\mathfrak{J}[t]^{\perp}$ for all $\left.\left.t \in\right] a, b\right]$, so that all $\mathcal{P}$-focal points are nondegenerate and, by $(2.3)$, the signature of a $\mathcal{P}$-focal point is equal to its multiplicity.

2.2. The differential problem. We consider the following setup. Let $g$ be a nondegenerate symmetric bilinear form in $\mathbb{R}^{n}$ and let $R:[a, b] \rightarrow \operatorname{Lin}\left(\mathbb{R}^{n}\right)$ be a continuous curve of linear endomorphisms of $\mathbb{R}^{n}$ such that $R(t)$ is $g$ symmetric for all $t$, i.e., $g(R(t) \cdot, \cdot)$ is a symmetric bilinear form on $\mathbb{R}^{n}$. Let $P \subset \mathbb{R}^{n}$ be a subspace on which $g$ is nondegenerate and let $S \in \operatorname{Bil}_{\text {sym }}(P)$ be a symmetric bilinear form on $P$. We will say that $(g, R, P, S)$ is an admissible quadruple for the differential problem in $\mathbb{R}^{n}$. We consider the linear differential equation in $\mathbb{R}^{n}$ :

$$
J^{\prime \prime}(t)=R(t) J(t), \quad t \in[a, b] ;
$$

we will consider solutions $J$ of (2.4) that satisfy in addition the following initial conditions:

$$
J(a) \in P,\left.\quad g\left(J^{\prime}(a)\right)\right|_{P}+S(J(a))=0 \in P^{*},
$$

where as usual we identify bilinear forms with linear maps taking values in the dual space. A solution $J$ of (2.4) satisfying the initial conditions (2.5) will be called a $(P, S)$-solution. Note that, if $P=\{0\}$ (and thus $S=0$ ), a $(P, S)$-solution is simply a solution of (2.4) vanishing at $t=a$. Let $\mathbb{J}$ denote the space of all $(P, S)$-solutions:

$$
\mathbb{J}=\{J: J \text { satisfies }(2.4) \text { and }(2.5)\} ;
$$

for $t \in[a, b]$, we set $\mathbb{J}[t]=\{J(t): J \in \mathbb{J}\}$. Observe that $\operatorname{dim}(\mathbb{J})=n$ because the pairs $\left(J(a), J^{\prime}(a)\right) \in \mathbb{R}^{n} \oplus \mathbb{R}^{n}$ satisfying (2.5) form an $n$-dimensional subspace. We say that an instant $t \in] a, b]$ is $(P, S)$-focal if there exists a nonzero $J \in \mathbb{J}$ with $J(t)=0$; the dimension of the space of those $J \in \mathbb{J}$ with $J(t)=0$ is called the multiplicity of $t$ and is denoted by $\operatorname{mul}(t)$. We have $\operatorname{mul}(t)=\operatorname{codim}(\mathbb{J}[t])=\operatorname{dim}\left(\mathbb{J}[t]^{\perp}\right)$, where $\perp$ denotes the orthogonal complement with respect to $g$. If $P=\{0\}$ then the $(P, S)$-focal instants are also called conjugate instants. The signature of a $(P, S)$-focal instant 
$t \in] a, b]$, denoted by $\operatorname{sgn}(t)$, is defined to be the signature of the restriction of $g$ to the space $\mathbb{J}[t]^{\perp}$; the $(P, S)$-focal instant $t$ is called nondegenerate if the restriction of $g$ to $\mathbb{J}[t]$ is nondegenerate. If the number of $(P, S)$-focal instants is finite we also define the focal index of the quadruple $(g, R, P, S)$ by $\mathrm{i}_{\text {foc }}(g, R, P, S)=\sum_{t \in] a, b]} \operatorname{sgn}(t)$. If $P=\{0\}$ then the focal index is also called the conjugate index.

Let $(\mathcal{M}, \mathfrak{g}, \gamma, \mathcal{P})$ be an admissible quadruple for the geometrical problem and denote by $n$ the dimension of $\mathcal{M}$. Consider a parallel referential along $\gamma$; such referential induces an isomorphism $\phi_{t}: T_{\gamma(t)} \mathcal{M} \rightarrow \mathbb{R}^{n}$ for all $t \in[a, b]$. Let $g$ be the nondegenerate symmetric bilinear form on $\mathbb{R}^{n}$ which equals the push-forward of $\mathfrak{g}_{\gamma(t)}$ by $\phi_{t}$; observe that, since $\mathfrak{g}$ is parallel, $g$ is independent of $t$. For each $t \in[a, b]$, let $R(t)$ denote the $g$-symmetric linear endomorphism of $\mathbb{R}^{n}$ such that $\phi_{t}^{-1} \circ R(t) \circ \phi_{t}$ equals the linear endomorphism $\mathcal{R}\left(\gamma^{\prime}(t), \cdot\right) \gamma^{\prime}(t)$ of $T_{\gamma(t)} \mathcal{M}$. Set $P=\phi_{a}\left(T_{\gamma(a)} \mathcal{P}\right)$ and let $S: P \times P \rightarrow \mathbb{R}$ be the push-forward of the second fundamental form $\mathcal{S}_{\gamma^{\prime}(a)}: T_{\gamma(a)} \mathcal{P} \times T_{\gamma(a)} \mathcal{P} \rightarrow \mathbb{R}$ by $\phi_{a}$. Then $(g, R, P, S)$ is an admissible quadruple for the differential problem in $\mathbb{R}^{n}$; we say that $(g, R, P, S)$ is obtained from $(\mathcal{M}, \mathfrak{g}, \gamma, \mathcal{P})$ by a parallel trivialization of the tangent bundle along $\gamma$. Obviously, the $\mathcal{P}$-focal points along $\gamma$ correspond to the $(P, S)$-focal instants of $(g, R, P, S)$; moreover, the multiplicity, signature and degeneracy of a $\mathcal{P}$-focal point along $\gamma$ are equal respectively to the multiplicity, signature and degeneracy of the corresponding $(P, S)$-focal instant. In particular, the quadruples $(\mathcal{M}, \mathfrak{g}, \gamma, \mathcal{P})$ and $(g, R, P, S)$ have the same focal indexes.

2.3. On the discreteness of the set of focal instants. We now give some sufficient conditions for the number of focal points along a semiRiemannian geodesic to be finite. We state our main results in terms of differential problems. Let $(g, R, P, S)$ be an admissible quadruple for the differential problem. A simple computation using the $g$-symmetry of $R$ shows that:

$$
g\left(J_{1}^{\prime}(t), J_{2}(t)\right)-g\left(J_{1}(t), J_{2}^{\prime}(t)\right) \equiv \text { constant },
$$

for all solutions $J_{1}, J_{2}$ of (2.4). The initial conditions (2.5) imply that the left-hand side of (2.7) vanish at $t=a$; hence:

$$
g\left(J_{1}^{\prime}(t), J_{2}(t)\right)=g\left(J_{1}(t), J_{2}^{\prime}(t)\right), \quad t \in[a, b],
$$

for all $J_{1}, J_{2} \in \mathbb{J}$. Observe that (2.8) and a dimension counting argument show that:

$$
\mathbb{J}[t]^{\perp}=\left\{J^{\prime}(t): J \in \mathbb{J}, J(t)=0\right\}, \quad t \in[a, b] .
$$

Proposition 2.2. If $(g, R, P, S)$ is an admissible quadruple for the differential problem then the nondegenerate $(P, S)$-focal instants are isolated. Moreover, there are no $(P, S)$-focal instants near $t=a$. 
Proof. Let $\left.\left.t_{0} \in\right] a, b\right]$ be a nondegenerate $(P, S)$-focal instant or let $t_{0}=a$. Since $\mathbb{J}[a]=P$ is nondegenerate for $g$, we have that, in any case, $\mathbb{J}\left[t_{0}\right]$ is nondegenerate for $g$. Denote by $e_{t}: \mathbb{J} \rightarrow \mathbb{R}^{n}$ the evaluation map $e_{t}(J)=$ $J(t)$. Choose a basis $\left(J_{i}\right)_{i=1}^{n}$ for $\mathbb{J}$ such that $\left(J_{i}\right)_{i=1}^{k}$ is a basis for the kernel of $e_{t_{0}}$; then $\left(J_{i}\left(t_{0}\right)\right)_{i=k+1}^{n}$ is a basis for $\mathbb{J}\left[t_{0}\right]$. Observe that an instant $t \in$ ]$a, b]$ is $(P, S)$-focal if and only if $e_{t}$ is not invertible, i.e., if and only if the determinant $\operatorname{det}\left(J_{i}(t)\right)_{i=1}^{n}$ is zero. Since a solution $J$ of $(2.4)$ is uniquely determined by the values of $J\left(t_{0}\right)$ and $J^{\prime}\left(t_{0}\right)$, equality (2.9) implies that the map $J \mapsto J^{\prime}\left(t_{0}\right)$ takes the kernel of $e_{t_{0}}$ isomorphically onto $\mathbb{J}\left[t_{0}\right]^{\perp}$. Thus $\left(J_{i}^{\prime}\left(t_{0}\right)\right)_{i=1}^{k}$ is a basis for $\mathbb{J}\left[t_{0}\right]^{\perp}$. For $i=1, \ldots, k$, define $\widetilde{J}_{i}:[a, b] \rightarrow \mathbb{R}^{n}$ by setting $\widetilde{J}_{i}(t)=\frac{1}{t-t_{0}} J_{i}(t)$ if $t \neq t_{0}$ and $\widetilde{J}_{i}\left(t_{0}\right)=J_{i}^{\prime}\left(t_{0}\right)$; since $J_{i}$ is of class $C^{2}$ and $J_{i}\left(t_{0}\right)=0$, it follows that $\widetilde{J}_{i}$ is of class $C^{1}$. For $i=k+1, \ldots, n$ we set $\widetilde{J}_{i}=J_{i}$ and then $\left(\widetilde{J}_{i}\left(t_{0}\right)\right)_{i=1}^{n}$ is a basis for $\mathbb{R}^{n}$, because the nondegeneracy of $g$ on $\mathbb{J}\left[t_{0}\right]$ implies $\mathbb{R}^{n}=\mathbb{J}\left[t_{0}\right] \oplus \mathbb{J}\left[t_{0}\right]^{\perp}$. It follows that $\operatorname{det}\left(J_{i}(t)\right)_{i=1}^{n}=$ $\left(t-t_{0}\right)^{k} \operatorname{det}\left(\widetilde{J}_{i}(t)\right)_{i=1}^{n}$ is nonzero for $t \neq t_{0}$ near $t_{0}$.

Remark 2.3. It is clear from the proof of Proposition 2.2 that the number of focal instants of a quadruple $(g, R, P, S)$ with $R$ real-analytic (or, similarly, the number of focal points corresponding to a quadruple $(\mathcal{M}, \mathfrak{g}, \gamma, \mathcal{P})$ with $(\mathcal{M}, \mathfrak{g})$ real-analytic) is finite.

\section{The Lagrangian Grassmannian and the Maslov index.}

Let $(V, \omega)$ be a symplectic space, i.e., $V$ is a finite-dimensional real vector space and $\omega$ is a symplectic form on $V$, i.e., a skew-symmetric nondegenerate symmetric bilinear form on $V$. A linear map $T: V \rightarrow V^{\prime}$ between symplectic spaces $(V, \omega),\left(V^{\prime}, \omega^{\prime}\right)$ is called a symplectomorphism if $T$ is an isomorphism and $\omega$ is the pull-back of $\omega^{\prime}$ by $T$. The set of all symplectomorphisms of $(V, \omega)$ is a closed Lie subgroup of the general linear group of $V$ and we will denote it by $\operatorname{Sp}(V, \omega)$. The Lie algebra of $\operatorname{Sp}(V, \omega)$, denoted by $\operatorname{sp}(V, \omega)$, consists of all linear endomorphisms $X: V \rightarrow V$ such that $\omega(X \cdot, \cdot)$ is a symmetric bilinear form on $V$. A symplectic space is necessarily even dimensional; we set $\operatorname{dim}(V)=2 n$. By a symplectic basis for $V$ we mean a basis $\left(b_{i}\right)_{i=1}^{2 n}$ of $V$ with $\omega\left(b_{i}, b_{n+i}\right)=-\omega\left(b_{n+i}, b_{i}\right)=1, i=1, \ldots, n$ and $\omega\left(b_{i}, b_{j}\right)=0$ otherwise. Every symplectic space admits a symplectic basis; in other words, every $2 n$ dimensional symplectic space is symplectomorphic to the space $\mathbb{R}^{n} \oplus \mathbb{R}^{n *}$ endowed with its canonical symplectic form:

$$
\omega\left(\left(v_{1}, \alpha_{1}\right),\left(v_{2}, \alpha_{2}\right)\right)=\alpha_{2}\left(v_{1}\right)-\alpha_{1}\left(v_{2}\right), \quad v_{1}, v_{2} \in \mathbb{R}^{n}, \alpha_{1}, \alpha_{2} \in \mathbb{R}^{n *} .
$$

The symplectic group of $\mathbb{R}^{n} \oplus \mathbb{R}^{n *}$ endowed with the symplectic form (3.1) will be denoted by $\operatorname{Sp}(2 n, \mathbb{R})$; its Lie algebra will be denoted by $\operatorname{sp}(2 n, \mathbb{R})$. If $\left(b_{i}\right)_{i=1}^{2 n}$ is a symplectic basis for $V$ and if a linear endomorphism $J: V \rightarrow V$ is defined by $J\left(b_{i}\right)=b_{n+i}, J\left(b_{n+i}\right)=-b_{i}, i=1, \ldots, n$ then $J$ is a complex 
structure for $V$ and the bilinear form $\omega(\cdot, J \cdot)$ is a positive definite inner product for $V$; we say that $J$ is compatible with $\omega$. Observe that $J$ is a symplectomorphism of $V$. If we identify the complex vector space $(V, J)$ with $\mathbb{C}^{n}$ by means of the complex basis $\left(b_{i}\right)_{i=1}^{n}$ then the symplectic form $\omega$ corresponds to minus the imaginary part of the canonical Hermitian product of $\mathbb{C}^{n}$. We will call the latter the canonical symplectic form of the space $\mathbb{C}^{n}$. Observe that the unitary group is contained in the symplectic group. A subspace $S \subset V$ is called isotropic if $\omega$ vanishes on $S$; an $n$-dimensional isotropic subspace of $V$ is called a Lagrangian subspace. We are interested in studying the $L a$ grangian Grassmannian of $(V, \omega)$, i.e., the set $\Lambda=\Lambda(V, \omega)$ of all Lagrangian subspaces of $V$. Obviously, symplectomorphisms map Lagrangian subspaces to Lagrangian subspaces, and therefore we get an action of $\operatorname{Sp}(V, \omega)$ on $\Lambda$; for every $T \in \operatorname{Sp}(V, \omega)$ we will still denote by $T$ the corresponding map $T: \Lambda \rightarrow \Lambda$. If $\mathbb{C}^{n}$ is endowed with its canonical symplectic form then a subspace $L \subset \mathbb{C}^{n}$ is Lagrangian if and only if it is a real form (i.e., $\mathbb{C}^{n}=L \oplus i L$ ) on which the canonical Hermitian product is real. It follows that the unitary group $\mathrm{U}(n)$ acts transitively in the Lagrangian Grassmannian of $\mathbb{C}^{n}$. The isotropy subgroup of the Lagrangian subspace $\mathbb{R}^{n} \subset \mathbb{C}^{n}$ is the orthogonal group $\mathrm{O}(n) \subset \mathrm{U}(n)$; in particular, the Lagrangian Grassmannian of $\mathbb{C}^{n}$ (and thus of any other $2 n$-dimensional symplectic space) is diffeomorphic to the homogeneous space $\mathrm{U}(n) / \mathrm{O}(n)$. Hence $\Lambda$ has the structure of a compact, connected, real-analytic $\frac{1}{2} n(n+1)$-dimensional manifold (it is indeed an embedded submanifold of the Grassmannian of all $n$-dimensional subspaces of $V)$.

If $J: V \rightarrow V$ is a complex structure compatible with $\omega$ and $L \subset V$ is a Lagrangian subspace then $J(L)$ is also Lagrangian and $V=L \oplus J(L)$. A pair of Lagrangians $\left(L_{0}, L_{1}\right)$ with $V=L_{0} \oplus L_{1}$ is called a Lagrangian decomposition for $V$. If $\left(L_{0}, L_{1}\right)$ is a Lagrangian decomposition for $V$ then the symplectic form $\omega$ induces a natural isomorphism between $L_{1}$ and the dual space of $L_{0}$. Namely, we define $\rho_{L_{0}, L_{1}}: L_{1} \rightarrow L_{0}^{*}$ by $\rho_{L_{0}, L_{1}}(v)=$ $\left.\omega(v, \cdot)\right|_{L_{0}}$, for all $v \in L_{1}$. The anti-symmetry of $\omega$ implies that $\rho_{L_{0}, L_{1}}=$ $-\rho_{L_{1}, L_{0}}^{*}$.

Remark 3.1. Observe that if $\left(b_{i}\right)_{i=1}^{n}$ is an arbitrary basis for $L_{0}$ and $\left(b_{i}\right)_{i=n+1}^{2 n}$ is the basis of $L_{1}$ which is mapped by $\rho_{L_{0}, L_{1}}$ to the dual basis of $\left(b_{i}\right)_{i=1}^{n}$ then $\left(b_{i}\right)_{i=1}^{2 n}$ is a symplectic basis of $V$. From this observation it follows that if $\left(L_{0}, L_{1}\right)$ and $\left(L_{0}^{\prime}, L_{1}^{\prime}\right)$ are Lagrangian decompositions then every isomorphism from $L_{0}$ to $L_{0}^{\prime}$ extends (uniquely) to a symplectomorphism of $V$ that carries $L_{1}$ to $L_{1}^{\prime}$. 
Given a Lagrangian subspace $L_{0} \subset V$, we consider the following subsets of $\Lambda$ :

$$
\begin{gathered}
\Lambda_{k}\left(L_{0}\right)=\left\{L \in \Lambda: \operatorname{dim}\left(L \cap L_{0}\right)=k\right\}, \quad k=0,1, \ldots, n ; \\
\Lambda_{\geq k}\left(L_{0}\right)=\bigcup_{j=k}^{n} \Lambda_{j}\left(L_{0}\right), \quad \Lambda_{\leq k}\left(L_{0}\right)=\bigcup_{j=0}^{k} \Lambda_{j}\left(L_{0}\right) .
\end{gathered}
$$

For $T \in \operatorname{Sp}(V, \omega)$, we have that $T\left(L_{0}\right)$ is in $\Lambda_{\leq k}\left(L_{0}\right)$ if and only if the linear map $L_{0} \ni v \mapsto T(v)+L_{0} \in V / L_{0}$ has rank greater than or equal to $n-k$; since this last condition is open, the set $\Lambda_{\leq k}\left(L_{0}\right)$ (and in particular the set $\left.\Lambda_{0}\left(L_{0}\right)\right)$ is open in $\Lambda$. Obviously the set $\Lambda_{\geq k}\left(L_{0}\right)$ is closed in $\Lambda$ and therefore $\Lambda_{k}\left(L_{0}\right)=\Lambda_{\geq k}\left(L_{0}\right) \cap \Lambda_{\leq k}\left(L_{0}\right)$ is locally closed, i.e., it is an open subset of its closure. This observation is used below to show that $\Lambda_{k}\left(L_{0}\right)$ is an embedded submanifold of $\Lambda$. We first need the following:

Lemma 3.2. Given Lagrangian subspaces $L_{0}, L_{1}, L_{0}^{\prime}, L_{1}^{\prime} \subset V$ with $\operatorname{dim}\left(L_{0} \cap\right.$ $\left.L_{1}\right)=\operatorname{dim}\left(L_{0}^{\prime} \cap L_{1}^{\prime}\right)$, there exists $T \in \operatorname{Sp}(V, \omega)$ with $T\left(L_{0}\right)=L_{0}^{\prime}$ and $T\left(L_{1}\right)=$ $L_{1}^{\prime}$.

Proof. By Remark 3.1 we may assume that $L_{0}=L_{0}^{\prime}$ and that $L_{0} \cap L_{1}=$ $L_{0}^{\prime} \cap L_{1}=S$. If $S^{\perp}$ denotes the $\omega$-orthogonal complement of the isotropic space $S$ then $S^{\perp} / S$ has a natural symplectic structure induced by $\omega$ and $\left(L_{0} / S, L_{1} / S\right),\left(L_{0} / S, L_{1}^{\prime} / S\right)$ are both Lagrangian decompositions of $S^{\perp} / S$. Again by Remark 3.1 we can find a symplectomorphism $\bar{T}$ of $S^{\perp} / S$ which is the identity on $L_{0} / S$ and such that $\bar{T}\left(L_{1} / S\right)=L_{1}^{\prime} / S$. Using a complementary Lagrangian to $L_{0}$ in $V$ it is easy to construct a symplectomorphism $T$ of $V$ that is the identity on $L_{0}$ and that induces $\bar{T}$ on the quotient $S^{\perp} / S$. Obviously $T\left(L_{1}\right)=L_{1}^{\prime}$.

Corollary 3.3. The set $\Lambda_{k}\left(L_{0}\right)$ is a locally closed orbit in $\Lambda$ of the Lie group $\operatorname{Sp}\left(V, \omega, L_{0}\right)$ of symplectomorphisms of $V$ that preserve $L_{0}$. In particular, $\Lambda_{k}\left(L_{0}\right)$ is an embedded submanifold of $\Lambda$.

Proof. Lemma 3.2 implies that $\Lambda_{k}\left(L_{0}\right)$ is an orbit of $\operatorname{Sp}\left(V, \omega, L_{0}\right)$ and a locally closed orbit of a Lie group is an embedded submanifold (see $[\mathbf{1 9}$, Theorem 2.9.7]).

Let us now describe an explicit differentiable atlas for $\Lambda$. Let $\left(L_{0}, L_{1}\right)$ be a Lagrangian decomposition for $V$; we define a map $\phi_{L_{0}, L_{1}}: \Lambda_{0}\left(L_{1}\right) \longrightarrow$ $\operatorname{Bil}_{\text {sym }}\left(L_{0}\right)$ by setting $\phi_{L_{0}, L_{1}}(L)(v, w)=\omega(Z v, w)$, for all $L \in \Lambda_{0}\left(L_{1}\right)$ and all $v, w \in L_{0}$, where $Z: L_{0} \rightarrow L_{1}$ is the unique linear map whose graph in $V=$ $L_{0} \oplus L_{1}$ equals $L$. By identifying $\operatorname{Bil}_{\text {sym }}\left(L_{0}\right)$ with a subspace of $\operatorname{Lin}\left(L_{0}, L_{0}^{*}\right)$, we have $\phi_{L_{0}, L_{1}}(L)=\rho_{L_{0}, L_{1}} \circ Z$. The map $\phi_{L_{0}, L_{1}}$ is a diffeomorphism onto $\operatorname{Bil}_{\text {sym }}\left(L_{0}\right)$, i.e., a chart on the manifold $\Lambda$. If $\left(L_{0}, L_{1}\right)$ and $\left(L_{0}, L_{1}^{\prime}\right)$ are Lagrangian decompositions for $V$ then an explicit computation of the transition map from $\phi_{L_{0}, L_{1}}$ to $\phi_{L_{0}, L_{1}^{\prime}}$ shows that $\mathrm{d} \phi_{L_{0}, L_{1}}\left(L_{0}\right)=\mathrm{d} \phi_{L_{0}, L_{1}^{\prime}}\left(L_{0}\right)$, i.e., 
the differential $\mathrm{d} \phi_{L_{0}, L_{1}}\left(L_{0}\right): T_{L_{0}} \Lambda \rightarrow \operatorname{Bil}_{\text {sym }}\left(L_{0}\right)$ does not depend on the choice of $L_{1}$. We shall henceforth identify the tangent space $T_{L} \Lambda$ with the space $\operatorname{Bil}_{\text {sym }}(L)$ by means of the isomorphism obtained by the differential of a chart of the form $\phi_{L, L_{1}}$. If $\ell: I \rightarrow \Lambda$ is a curve of class $C^{1}$ defined in an interval $I$ then the derivative $\ell^{\prime}\left(t_{0}\right) \in \operatorname{Bil}_{\text {sym }}\left(\ell\left(t_{0}\right)\right), t_{0} \in I$, can be computed as follows; choose $v, w \in \ell\left(t_{0}\right)$ and a map $\mathfrak{v}:\left(t_{0}-\varepsilon, t_{0}+\varepsilon\right) \rightarrow V$ of class $C^{1}$ with $\mathfrak{v}\left(t_{0}\right)=v$ and $\mathfrak{v}(t) \in \ell(t)$ for all $t$. Then $\ell^{\prime}\left(t_{0}\right)(v, w)=\omega\left(\mathfrak{v}^{\prime}\left(t_{0}\right), w\right)$. Using this formula one can easily prove the following:

Lemma 3.4. Given a Lagrangian $L_{0} \in \Lambda$, denote by $\beta_{L_{0}}: \operatorname{Sp}(V, \omega) \rightarrow \Lambda$ the evaluation map $\beta_{L_{0}}(T)=T\left(L_{0}\right), T \in \operatorname{Sp}(V, \omega)$; its differential is given by:

$$
\mathrm{d} \beta_{L_{0}}(T) \cdot(X T)=\left.\omega(X \cdot, \cdot)\right|_{T\left(L_{0}\right)} \in \operatorname{Bil}_{\text {sym }}\left(T\left(L_{0}\right)\right),
$$

for all $X \in \operatorname{sp}(V, \omega)$. If $T \in \operatorname{Sp}(V, \omega)$ is a symplectomorphism then the differential of the induced diffeomorphism $T: \Lambda \rightarrow \Lambda$ is given by:

$$
\mathrm{d} T(L) \cdot B=\left.B\left(T^{-1} \cdot, T^{-1} \cdot\right)\right|_{T(L)} \in \operatorname{Bil}_{\mathrm{sym}}(T(L)),
$$

for all $L \in \Lambda, B \in \operatorname{Bil}_{\text {sym }}(L)$. Given a Lagrangian decomposition $\left(L_{0}, L_{1}\right)$ then the differential of the chart $\phi_{L_{0}, L_{1}}$ at a point $L \in \Lambda_{0}\left(L_{1}\right)$ is given by:

$$
\mathrm{d} \phi_{L_{0}, L_{1}}(L) \cdot B=\left.B(\eta \cdot, \eta \cdot)\right|_{L_{0}} \in \operatorname{Bil}_{\mathrm{sym}}\left(L_{0}\right), \quad B \in \operatorname{Bil}_{\mathrm{sym}}(L),
$$

where the isomorphism $\eta: L_{0} \rightarrow L$ is the restriction to $L_{0}$ of the projection onto $L$ relative to the decomposition $V=L \oplus L_{1}$.

Using Formula (3.2) it is easy to compute the tangent space of the manifold $\Lambda_{k}\left(L_{0}\right)$ at a point $L \in \Lambda_{k}\left(L_{0}\right)$. Namely, if $\operatorname{sp}\left(V, \omega, L_{0}\right)$ denotes the Lie algebra of $\operatorname{Sp}\left(V, \omega, L_{0}\right)$ then Corollary 3.3 implies that the tangent space $T_{L} \Lambda_{k}\left(L_{0}\right)$ is equal to the image of $\operatorname{sp}\left(V, \omega, L_{0}\right)$ by $\mathrm{d} \beta_{L}(\mathrm{Id})$; keeping in mind that $\operatorname{sp}\left(V, \omega, L_{0}\right)$ consists of those linear endomorphisms $X: V \rightarrow V$ such that $\omega(X \cdot, \cdot)$ is a symmetric bilinear form on $V$ vanishing on $L_{0}$, we obtain from (3.2) that $T_{L} \Lambda_{k}\left(L_{0}\right)$ equals the space of bilinear forms $B \in \operatorname{Bil}_{\text {sym }}(L)$ that vanish on $L \cap L_{0}$. Thus, the restriction map $\left.B \mapsto B\right|_{L \cap L_{0}}$ induces an isomorphism between the quotient $T_{L} \Lambda / T_{L} \Lambda_{k}\left(L_{0}\right)$ and $\operatorname{Bil}_{\text {sym }}\left(L \cap L_{0}\right)$; in particular, $\Lambda_{k}\left(L_{0}\right)$ has codimension $\frac{1}{2} k(k+1)$ in $\Lambda$ and $\Lambda_{1}\left(L_{0}\right)$ is a hypersurface on $\Lambda$. Such hypersurface has a canonical transverse orientation which is defined by declaring a vector $B \in T_{L} \Lambda, B \notin T_{L} \Lambda_{1}\left(L_{0}\right)$, positively oriented if the nonzero symmetric bilinear form $\left.B\right|_{L \cap L_{0}}$ on the line $L \cap L_{0}$ is positive definite.

Remark 3.5. If $\left\{L_{j}: j=1,2, \ldots\right\}$ is a countable subset of $\Lambda$ then we can find a Lagrangian $L \in \Lambda$ with $L \cap L_{j}=\{0\}$ for all $j$. Namely, we have shown above that $\Lambda_{k}\left(L_{j}\right)$ is a submanifold of $\Lambda$ of positive codimension for $k=1, \ldots, n$; hence, the union $\bigcup_{j=1}^{+\infty} \bigcup_{k=1}^{n} \Lambda_{k}\left(L_{j}\right)$ has null measure in $\Lambda$. 
Let $L \in \Lambda$ in the domain of $\phi_{L_{0}, L_{1}}$ be fixed and set $\beta=\phi_{L_{0}, L_{1}}(L) \in$ $\operatorname{Bil}_{\text {sym }}\left(L_{0}\right)$. Then:

$$
L_{0} \cap L=\operatorname{Ker}(\beta)
$$

then $L \in \Lambda_{k}\left(L_{0}\right)$ iff the kernel of $\beta$ has dimension equal to $k$ and in particular $L \in \Lambda_{\geq 1}\left(L_{0}\right)$ iff $\beta$ is degenerate. Assume now that $\ell: I \subset \mathbb{R} \rightarrow \Lambda$ is a curve of class $C^{1}$ in $\Lambda$ with image in the domain of $\phi_{L_{0}, L_{1}}$; set $\beta=\phi_{L_{0}, L_{1}} \circ \ell$. Using Formula (3.3) and keeping in mind that the isomorphism $\eta$ in (3.3) is the identity on $L_{0} \cap L$ we conclude that:

$$
\left.\ell^{\prime}\left(t_{0}\right)\right|_{\ell\left(t_{0}\right) \cap L_{0}}=\left.\beta^{\prime}\left(t_{0}\right)\right|_{\operatorname{Ker}\left(\beta\left(t_{0}\right)\right)},
$$

for all $t_{0} \in I$. We are now going to define the Maslov index of a curve of Lagrangians. We start by recalling from [1] that the fundamental group of the Lagrangian Grassmannian is infinite cyclic. More explicitly, we have $\Lambda \cong \mathrm{U}(n) / \mathrm{O}(n)$; the square of the determinant map induces a fibration $\operatorname{det}^{2}: \mathrm{U}(n) / \mathrm{O}(n) \rightarrow S^{1}$. The long exact homotopy sequence of such fibration shows that the map $\operatorname{det}^{2}$ induces an isomorphism between fundamental groups; then, by Hurewicz theorem, $\pi_{1}(\Lambda) \cong H_{1}(\Lambda) \cong \mathbb{Z}$. Let $L_{0} \in \Lambda$ be fixed. The open set $\Lambda_{0}\left(L_{0}\right) \subset \Lambda$ is contractible (because it is diffeomorphic to a vector space) and therefore the inclusion of $(\Lambda, \emptyset)$ in $\left(\Lambda, \Lambda_{0}\left(L_{0}\right)\right)$ induces an isomorphism in singular homology. So we have an isomorphism:

$$
\mu_{L_{0}}: H_{1}\left(\Lambda, \Lambda_{0}\left(L_{0}\right)\right) \stackrel{\cong}{\longrightarrow} \mathbb{Z} .
$$

The Maslov index (relative to $L_{0}$ ) of a continuous curve $\ell:[a, b] \rightarrow \Lambda$ with endpoints in $\Lambda_{0}\left(L_{0}\right)$ is defined as the integer number corresponding to the homology class determined by $\ell$ in $H_{1}\left(\Lambda, \Lambda_{0}\left(L_{0}\right)\right)$ via the isomorphism (3.6). We denote the Maslov index of $\ell$ by $\mu_{L_{0}}(\ell)$. Obviously the Maslov index is additive by concatenation and invariant by homotopies of curves with endpoints in $\Lambda_{0}\left(L_{0}\right)$. Actually, in order to complete the definition of Maslov index we have to choose a sign for the isomorphism (3.6) (Lemma 3.8 below).

Lemma 3.6. Let $\left(L_{0}, L_{1}\right)$ be a Lagrangian decomposition for $V$ and let $\ell_{1}, \ell_{2}:[a, b] \rightarrow \Lambda$ be continuous curves with endpoints in $\Lambda_{0}\left(L_{0}\right)$ and image contained in the domain of the chart $\phi_{L_{0}, L_{1}}$; set $\beta_{i}=\phi_{L_{0}, L_{1}} \circ \ell_{i}:[a, b] \rightarrow$ $\operatorname{Bil}_{\text {sym }}\left(L_{0}\right), i=1,2$. If $n_{+}\left(\beta_{1}(a)\right)=n_{+}\left(\beta_{2}(a)\right)$ and $n_{+}\left(\beta_{1}(b)\right)=n_{+}\left(\beta_{2}(b)\right)$ then the curves $\ell_{1}$ and $\ell_{2}$ are homologous in $H_{1}\left(\Lambda, \Lambda_{0}\left(L_{0}\right)\right)$.

Proof. Since the set of nondegenerate symmetric bilinear forms on $L_{0}$ with a fixed coindex is connected, we can find a homotopy from $\beta_{1}$ to $\beta_{2}$ through curves in $\operatorname{Bil}_{\text {sym }}\left(L_{0}\right)$ whose endpoints are nondegenerate symmetric bilinear forms. Such homotopy lifts via $\phi_{L_{0}, L_{1}}$ to a homotopy from $\ell_{1}$ to $\ell_{2}$ through curves with endpoints in $\Lambda_{0}\left(L_{0}\right)$ (recall (3.4)).

Lemma 3.7. Let $\beta$ : $\left[t_{0}, t_{1}\left[\rightarrow \operatorname{Bil}_{\mathrm{sym}}\left(\mathbb{R}^{n}\right)\right.\right.$ be a $C^{1}$ curve such that the restriction of $\beta^{\prime}\left(t_{0}\right)$ to the kernel of $\beta\left(t_{0}\right)$ is nondegenerate. Then, for $\varepsilon>0$ 
sufficiently small, the bilinear form $\beta\left(t_{0}+\varepsilon\right)$ is nondegenerate and its coindex is given by:

$$
n_{+}\left(\beta\left(t_{0}+\varepsilon\right)\right)=n_{+}\left(\beta\left(t_{0}\right)\right)+n_{+}\left(\left.\beta^{\prime}\left(t_{0}\right)\right|_{\operatorname{Ker}\left(\beta\left(t_{0}\right)\right)}\right) .
$$

Thus, if $\beta$ is of class $C^{1}$ in a neighborhood of $t_{0}$ then $\beta(t)$ is nondegenerate for $t \neq t_{0}$ sufficiently near $t_{0}$ and:

$$
n_{+}\left(\beta\left(t_{0}+\varepsilon\right)\right)-n_{+}\left(\beta\left(t_{0}-\varepsilon\right)\right)=\operatorname{sgn}\left(\left.\beta^{\prime}\left(t_{0}\right)\right|_{\operatorname{Ker}\left(\beta\left(t_{0}\right)\right)}\right),
$$

for $\varepsilon>0$ sufficiently small.

Proof. See [12, Proposition 2.5].

The lemma below gives a canonical sign for the isomorphism (3.6).

Lemma 3.8. Let $\ell:[a, b] \rightarrow \Lambda$ be a curve of class $C^{1}$ such that:

(a) $\ell(t) \in \Lambda_{\geq 1}\left(L_{0}\right)$ at a unique instant $\left.t=t_{0} \in\right] a, b[$;

(b) $\ell\left(t_{0}\right) \in \Lambda_{1}\left(L_{0}\right)$;

(c) $\ell^{\prime}\left(t_{0}\right)$ is positively oriented in $T_{\ell\left(t_{0}\right)} \Lambda / T_{\ell\left(t_{0}\right)} \Lambda_{1}\left(L_{0}\right)$.

Then $\ell$ defines a generator of $H_{1}\left(\Lambda, \Lambda_{0}\left(L_{0}\right)\right)$. Moreover, any two curves of class $C^{1}$ in $\Lambda$ satisfying conditions (a), (b), (c) are homologous in $H_{1}(\Lambda$, $\left.\Lambda_{0}\left(L_{0}\right)\right)$.

Proof. Recalling that $\operatorname{det}^{2}: \Lambda \cong \mathrm{U}(n) / \mathrm{O}(n) \rightarrow S^{1}$ induces an isomorphism between fundamental groups it is easy to exhibit a generator of the fundamental group of $\Lambda$ (and hence of $H_{1}\left(\Lambda, \Lambda_{0}\left(L_{0}\right)\right)$ ) that satisfies (a), (b) and (c). We now have to show that if $\ell_{1}, \ell_{2}:\left[t_{0}-\varepsilon, t_{0}+\varepsilon\right] \rightarrow \Lambda$ are curves of class $C^{1}$ in $\Lambda$ satisfying (a), (b) and (c) then $\ell_{1}$ and $\ell_{2}$ are homologous in $H_{1}\left(\Lambda, \Lambda_{0}\left(L_{0}\right)\right)$. The action of $\operatorname{Sp}\left(V, \omega, L_{0}\right)$ preserves the transverse orientation of $\Lambda_{1}\left(L_{0}\right)$ in $\Lambda$ and it induces the identity ${ }^{1}$ on $H_{1}\left(\Lambda, \Lambda_{0}\left(L_{0}\right)\right)$. Since $\operatorname{Sp}\left(V, \omega, L_{0}\right)$ acts transitively on $\Lambda_{1}\left(L_{0}\right)$ (Corollary 3.3), we may assume that $\ell_{1}\left(t_{0}\right)=\ell_{2}\left(t_{0}\right)=L$. The conclusion follows from Lemmas 3.6, 3.7 and Formulas (3.4) and (3.5), using a local chart $\phi_{L_{0}, L_{1}}$ around $L$ (the existence of such chart follows from Remark 3.5).

From now on, we fix the sign of the isomorphism (3.6) by choosing any $C^{1}$ curve in $\Lambda$ satisfying (a), (b) and (c) as a generator of $H_{1}\left(\Lambda, \Lambda_{0}\left(L_{0}\right)\right)$.

Proposition 3.9. Let $\ell:[a, b] \rightarrow \Lambda$ be a continuous curve with endpoints outside $\Lambda_{\geq 1}\left(L_{0}\right)$. If there exists a Lagrangian subspace $L_{1}$ complementary to $L_{0}$ and such that the image of $\ell$ is entirely contained in the domain $\Lambda_{0}\left(L_{1}\right)$

\footnotetext{
${ }^{1} \operatorname{Since} \operatorname{Sp}(V, \omega)$ is connected, for $T \in \operatorname{Sp}(V, \omega)$, the diffeomorphism $T: \Lambda \rightarrow \Lambda$ is homotopic to the identity of $\Lambda$ and therefore induces the identity of $H_{1}(\Lambda)$. It follows from functoriality of homology that every $T \in \operatorname{Sp}\left(V, \omega, L_{0}\right)$ induces the identity on $H_{1}\left(\Lambda, \Lambda_{0}\left(L_{0}\right)\right)$.
} 
of the chart $\phi_{L_{0}, L_{1}}$ then, setting $\beta=\phi_{L_{0}, L_{1}} \circ \ell$, the Maslov index of $\ell$ with respect to $L_{0}$ is given by:

$$
\mu_{L_{0}}(\ell)=n_{+}(\beta(b))-n_{+}(\beta(a)) .
$$

Proof. By Lemma 3.6 it suffices to find for every $i, j=0, \ldots, n$ a continuous curve $\widetilde{\beta}:[0,1] \rightarrow \operatorname{Bil}_{\text {sym }}\left(L_{0}\right)$ such that the Maslov index of $\widetilde{\ell}=\phi_{L_{0}, L_{1}}^{-1} \circ \widetilde{\beta}$ with respect to $L_{0}$ is equal to $j-i$ and such that $\widetilde{\beta}(0), \widetilde{\beta}(1)$ are both nondegenerate and have coindexes respectively equal to $i$ and $j$. It is easy to provide examples of such a curve $\widetilde{\beta}$ by considering, for instance, diagonal matrices.

Corollary 3.10. Let $\ell:[a, b] \rightarrow \Lambda$ be a curve of class $C^{1}$ having endpoints outside $\Lambda_{\geq 1}\left(L_{0}\right)$. If for all $\left.t \in\right] a, b\left[\right.$ such that $\ell(t) \in \Lambda_{\geq 1}\left(L_{0}\right)$ we have that $\ell^{\prime}(t)$ is nondegenerate on $\ell(t) \cap L_{0}$, then the number of intersections of $\ell$ with $\Lambda_{\geq 1}\left(L_{0}\right)$ is finite, and:

$$
\mu_{L_{0}}(\ell)=\sum_{t \in] a, b[} \operatorname{sgn}\left(\left.\ell^{\prime}(t)\right|_{\ell(t) \cap L_{0}}\right) .
$$

Proof. If $\ell(t) \in \Lambda_{\geq 1}\left(L_{0}\right)$ then, by Remark 3.5, we can find a Lagrangian $L_{1} \in$ $\Lambda$ with $L_{1} \cap L_{0}=L_{1} \cap \ell(t)=\{0\}$; thus we can consider the local chart $\phi_{L_{0}, L_{1}}$ around $\ell(t)$. Keeping in mind Formulas (3.4) and (3.5), it follows directly from Proposition 3.9 and Lemma 3.7 that $\mu_{L_{0}}\left(\left.\ell\right|_{[t-\varepsilon, t+\varepsilon]}\right)=\operatorname{sgn}\left(\left.\ell^{\prime}(t)\right|_{\ell(t) \cap L_{0}}\right)$ for $\varepsilon>0$ small enough. The conclusion now follows from the additivity of the Maslov index by concatenation of curves.

\section{Applications of the Maslov index: Stability of the focal index.}

4.1. The Maslov index of a differential problem. Let $(g, R, P, S)$ be an admissible quadruple for the differential problem. By setting $\alpha(t)=$ $g J^{\prime}(t) \in \mathbb{R}^{n *}$, the second order differential equation (2.4) can be rewritten as:

$$
\frac{\mathrm{d}}{\mathrm{d} t}\left(\begin{array}{c}
J(t) \\
\alpha(t)
\end{array}\right)=X(t)\left(\begin{array}{c}
J(t) \\
\alpha(t)
\end{array}\right)
$$

where $X(t)$ is the linear endomorphism of $\mathbb{R}^{n} \oplus \mathbb{R}^{n *}$ given by:

$$
X(t)=\left(\begin{array}{cc}
0 & g^{-1} \\
g R(t) & 0
\end{array}\right), \quad t \in[a, b] .
$$

Let $\Phi$ denote the fundamental matrix of the system (4.1), i.e., for each $t \in[a, b], \Phi(t)$ is the isomorphism of $\mathbb{R}^{n} \oplus \mathbb{R}^{n *}$ such that:

$$
\Phi(t)\left(J(a), g J^{\prime}(a)\right)=\left(J(t), g J^{\prime}(t)\right),
$$


for all solutions $J$ of (2.4). Then $\Phi$ satisfies the following matrix differential equation:

$$
\Phi^{\prime}(t)=X(t) \Phi(t), \quad t \in[a, b],
$$

with initial condition $\Phi(a)=\mathrm{Id}$. The conservation law (2.7) implies that $\Phi(t)$ is indeed a symplectomorphism of the symplectic space $\mathbb{R}^{n} \oplus \mathbb{R}^{n *}$ endowed with its canonical symplectic form (3.1); alternatively, the fact that $\Phi(t)$ belongs to $\operatorname{Sp}(2 n, \mathbb{R})$ follows from (4.4) by observing that $X(t)$ belongs to the Lie algebra $\operatorname{sp}(2 n, \mathbb{R})$.

Consider the following two Lagrangian subspaces of $\mathbb{R}^{n} \oplus \mathbb{R}^{n *}$ :

$$
\begin{gathered}
\ell_{0}=\left\{(v, \alpha) \in \mathbb{R}^{n} \oplus \mathbb{R}^{n *}: v \in P,\left.\alpha\right|_{P}+S(v)=0 \in P^{*}\right\}, \\
L_{0}=\{0\} \oplus \mathbb{R}^{n *} ;
\end{gathered}
$$

observe that the initial condition (2.5) is equivalent to $\left(J(a), g J^{\prime}(a)\right) \in \ell_{0}$. Since $\Phi(t)$ is a symplectomorphism, the space $\ell(t)=\Phi(t)\left(\ell_{0}\right)$ is Lagrangian; more explicitly, $\ell(t)$ is given by:

$$
\ell(t)=\left\{\left(J(t), g J^{\prime}(t)\right): J \in \mathbb{J}\right\},
$$

for all $t \in[a, b]$. From (2.9) it follows that:

$$
\ell(t) \cap L_{0}=\{0\} \oplus \mathbb{J}[t]^{\circ} \subset\{0\} \oplus \mathbb{R}^{n *} .
$$

In particular, an instant $t \in] a, b]$ is $(P, S)$-focal if and only if $\ell(t) \cap L_{0} \neq\{0\}$; moreover, $t$ has multiplicity $k$ if and only if $\ell(t) \in \Lambda_{k}\left(L_{0}\right)$. In the notation of Lemma 3.4, we have:

$$
\ell=\beta_{\ell_{0}} \circ \Phi,
$$

and in particular $\ell$ is a curve of class $C^{1}$ on the Lagrangian Grassmannian $\Lambda$ of $\mathbb{R}^{n} \oplus \mathbb{R}^{n *}$. Recalling Proposition 2.2 , we give the following:

Definition 4.1. Let $(g, R, P, S)$ be an admissible quadruple for the differential problem and assume that $t=b$ is not a $(P, S)$-focal instant. The Maslov index of $(g, R, P, S)$ is defined by $\mathrm{i}_{\text {Maslov }}(g, R, P, S)=\mu_{L_{0}}\left(\left.\ell\right|_{[a+\varepsilon, b]}\right)$, where $\varepsilon>0$ is chosen in such a way that there are no $(P, S)$-focal instants in $] a, a+\varepsilon]$.

Obviously the Maslov index of $(g, R, P, S)$ does not depend on the choice of $\varepsilon$.

We now want to compute the Maslov index of a quadruple $(g, R, P, S)$ using Corollary 3.10. We start by computing the derivative $\ell^{\prime}(t)$ in the lemma below.

Lemma 4.2. For every $t \in[a, b]$ the isomorphism $\mathbb{J}[t]^{\perp} \ni v \mapsto(0, g(v)) \in$ $\ell(t) \cap L_{0}$ carries the restriction of $g$ to the restriction of $\ell^{\prime}(t)$. 
Proof. From (3.2), (4.4) and (4.9) it follows that:

$$
\ell^{\prime}(t)=\left.\omega(X(t) \cdot, \cdot)\right|_{\ell(t)} .
$$

The conclusion then follows from (4.2) and (4.8).

Theorem 4.3. Let $(g, R, P, S)$ be an admissible quadruple for the differential problem in $\mathbb{R}^{n}$. If all the $(P, S)$-focal instants are nondegenerate and if $t=b$ is not $(P, S)$-focal then the focal index of $(g, R, P, S)$ is (welldefined and) equal to the Maslov index of $(g, R, P, S)$, i.e., $\mathrm{i}_{\text {foc }}(g, R, P, S)=$ $\mathrm{i}_{\text {Maslov }}(g, R, P, S)$.

Proof. Follows directly from Corollary 3.10 and Lemma 4.2.

Corollary 4.4. Let $(\mathcal{M}, \mathfrak{g}, \mathcal{P}, \gamma)$ be an admissible quadruple for the geometric problem such that $\gamma(b)$ is not a $\mathcal{P}$-focal point. Assume that $(\mathcal{M}, \mathfrak{g})$ is Riemannian or Lorentzian, and in the latter case, that $\gamma$ is nonspacelike. If $(g, R, P, S)$ corresponds to $(\mathcal{M}, \mathfrak{g}, \mathcal{P}, \gamma)$ by a parallel trivialization of the tangent bundle then the Maslov index of $(g, R, P, S)$ is equal to the sum of the multiplicities of the $\mathcal{P}$-focal points, i.e., $\mathrm{i}_{\text {Maslov }}(g, R, P, S)=\sum_{t \in] a, b]} \operatorname{mul}(t)$.

Remark 4.5. It is easy to see that every Lagrangian subspace $\ell_{0} \subset \mathbb{R}^{n} \oplus$ $\mathbb{R}^{n *}$ is of the form (4.5) for a unique pair $(P, S)$, with $P \subset \mathbb{R}^{n}$ a subspace and $S: P \times P \rightarrow \mathbb{R}$ a symmetric bilinear form. This means that (2.5) is the most general form of initial conditions that yield a Lagrangian subspace.

4.2. Stability of the indexes. Let $G_{r}(n) \cong \mathrm{O}(n) /(\mathrm{O}(r) \times \mathrm{O}(n-r))$ denote the Grassmannian of all $r$-dimensional subspaces of $\mathbb{R}^{n}$. Let $E_{r, n}$ denote the tautological vector bundle over $G_{r}(n)$, i.e., the set of pairs $(P, v) \in G_{r}(n) \times \mathbb{R}^{n}$ with $v \in P$. We denote by $\bigvee_{2} E_{r, n}^{*}$ the second symmetric power of the dual bundle of $E_{r, n}$, i.e., $\bigvee_{2} E_{r, n}^{*}$ is a vector bundle over the Grassmannian $G_{r}(n)$ whose fiber over $P \in G_{r}(n)$ is the $\operatorname{space} \operatorname{Bil}_{\text {sym }}(P)$ of symmetric bilinear forms on $P$. The elements of $\bigvee_{2} E_{r, n}^{*}$ are thus pairs $(P, S)$ with $P \subset \mathbb{R}^{n}$ an $r$-dimensional subspace and $S: P \times P \rightarrow \mathbb{R}$ a symmetric bilinear form. We have a bijective map (see Remark 4.5):

$$
\bigvee_{2} E_{r, n}^{*} \ni(P, S) \longmapsto \ell_{0} \in \Lambda_{n-r}\left(L_{0}\right),
$$

where $\ell_{0}$ is defined as in (4.5). The map (4.11) is in fact a smooth diffeomorphism. This can be proven, for instance, by observing that the Lie group $\operatorname{Sp}\left(2 n, \mathbb{R}, L_{0}\right)$ of symplectomorphisms of $\mathbb{R}^{n} \oplus \mathbb{R}^{n *}$ that preserve $L_{0}$ acts smoothly and transitively in both $\bigvee_{2} E_{r, n}^{*}$ and $\Lambda_{n-r}\left(L_{0}\right)$; moreover, the map (4.11) intertwines such actions.

We can now prove the following result about the stability of the Maslov and the focal indexes for quadruples $(g, R, P, S)$.

Theorem 4.6. For each $j \in \mathbb{N} \cup\{\infty\}$, let $\left(g_{j}, R_{j}, P_{j}, S_{j}\right)$ be an admissible quadruple for the differential problem in $\mathbb{R}^{n}$. 
Assume that $\left(g_{j}, R_{j}, P_{j}, S_{j}\right)$ converges to $\left(g_{\infty}, R_{\infty}, P_{\infty}, S_{\infty}\right)$ in the following sense:

(1) $\operatorname{dim}\left(P_{j}\right)=r$ for all $j \in \mathbb{N} \cup\{\infty\}$ and some $r=0, \ldots, n$;

(2) $\left(P_{j}, S_{j}\right) \rightarrow\left(P_{\infty}, S_{\infty}\right)$ in $\bigvee_{2} E_{r, n}^{*}$ as $j \rightarrow \infty$;

(3) $g_{j} \rightarrow g_{\infty}$ in $\operatorname{Bil}_{\text {sym }}\left(\mathbb{R}^{n}\right)$ as $j \rightarrow \infty$;

(4) $R_{j} \rightarrow R_{\infty}$ uniformly on $[a, b]$ as $j \rightarrow \infty$.

If $t_{0}=b$ is not $\left(P_{\infty}, S_{\infty}\right)$-focal for $\left(g_{\infty}, R_{\infty}, P_{\infty}, S_{\infty}\right)$ then, for $j \in \mathbb{N}$ sufficiently large, $t_{0}=b$ is not $\left(P_{j}, S_{j}\right)$-focal for $\left(g_{j}, R_{j}, P_{j}, S_{j}\right)$, and:

$$
\mathrm{i}_{\text {Maslov }}\left(g_{j}, R_{j}, P_{j}, S_{j}\right)=\mathrm{i}_{\text {Maslov }}\left(g_{\infty}, R_{\infty}, P_{\infty}, S_{\infty}\right) .
$$

In particular, if all the $\left(P_{j}, S_{j}\right)$-focal instants of $\left(g_{j}, R_{j}, P_{j}, S_{j}\right)$ are nondegenerate for all $j \in \mathbb{N} \cup\{\infty\}$ then, for $j$ sufficiently large:

$$
\mathrm{i}_{\text {foc }}\left(g_{j}, R_{j}, P_{j}, S_{j}\right)=\mathrm{i}_{\text {foc }}\left(g_{\infty}, R_{\infty}, P_{\infty}, S_{\infty}\right) .
$$

Proof. We define objects $\left(\ell_{0}\right)_{j}, \Phi_{j}$ and $\ell_{j}$ corresponding to the quadruple $\left(g_{j}, R_{j}, P_{j}, S_{j}\right)$ in analogy respectively with (4.5), (4.3) and (4.7). Since $\left(P_{j}, S_{j}\right) \rightarrow(P, S)$, the fact that (4.11) is a diffeomorphism implies that $\left(\ell_{0}\right)_{j} \rightarrow \ell_{0}$ in $\Lambda$. Standard results on the continuous dependence of solutions of a differential equation with respect to the data show that $\Phi_{j} \rightarrow \Phi$ uniformly on $[a, b]$ (and even in the $C^{1}$-topology). The smoothness of the action of $\operatorname{Sp}(2 n, \mathbb{R})$ on $\Lambda$ and (4.9) imply then that $\ell_{j} \rightarrow \ell$ on the compactopen topology; in particular, $\ell_{j}(b) \rightarrow \ell(b)$ in $\Lambda$. The fact that $t_{0}=b$ is not $\left(P_{\infty}, S_{\infty}\right)$-focal for $\left(g_{\infty}, R_{\infty}, P_{\infty}, S_{\infty}\right)$ means that $\ell(b)$ belongs to the open set $\Lambda_{0}\left(L_{0}\right)$; thus $\ell_{j}(b) \in \Lambda_{0}\left(L_{0}\right)$ for large $j$ and therefore $t_{0}=b$ is not $\left(P_{j}, S_{j}\right)$-focal for $\left(g_{j}, R_{j}, P_{j}, S_{j}\right)$ as well. We will see in Lemma 4.10 below that there exists $\varepsilon>0$ such that none of the quadruples $\left(g_{j}, R_{j}, P_{j}, S_{j}\right)$ has focal instants in $] a, a+\varepsilon]$; thus:

$$
\mathrm{i}_{\text {Maslov }}\left(g_{j}, R_{j}, P_{j}, S_{j}\right)=\mu_{L_{0}}\left(\left.\ell_{j}\right|_{[a+\varepsilon, b]}\right), \quad \mathrm{i}_{\text {Maslov }}(g, R, P, S)=\mu_{L_{0}}\left(\left.\ell\right|_{[a+\varepsilon, b]}\right) .
$$

Since $\Lambda$ is semi-locally simply-connected and $\Lambda_{0}\left(L_{0}\right)$ is locally arc-connected, the fact that $\ell_{j} \rightarrow \ell$ in the compact-open topology implies that $\left.\ell_{j}\right|_{[a+\varepsilon, b]}$ is homologous to $\left.\ell\right|_{[a+\varepsilon, b]}$ in $H_{1}\left(\Lambda, \Lambda_{0}\left(L_{0}\right)\right)$ for $j$ sufficiently large; thus $\mu_{L_{0}}\left(\left.\ell_{j}\right|_{[a+\varepsilon, b]}\right)=\mu_{L_{0}}\left(\ell_{[a+\varepsilon, b]}\right)$ for such $j$. This proves equality (4.12); equality (4.13) now follows from Theorem 4.3.

If $(\mathcal{M}, \mathfrak{g})$ is an $n$-dimensional semi-Riemannian manifold and $\mathcal{P} \subset \mathcal{M}$ is a smooth submanifold then the normal bundle $T \mathcal{P}^{\perp}=\bigcup_{p \in \mathcal{P}} T_{p} \mathcal{P}^{\perp}$ is a smooth $n$-dimensional submanifold of the tangent bundle $T \mathcal{M}$. Thus, for every $\mathfrak{n} \in T_{p} \mathcal{P}^{\perp}, p \in \mathcal{P}$, the tangent space $T_{\mathfrak{n}}\left(T \mathcal{P}^{\perp}\right)$ is an $n$-dimensional subspace of the $2 n$-dimensional space $T_{\mathfrak{n}} T \mathcal{M}$. Moreover, if $\mathcal{P}$ is nondegenerate at $p$ and if one identifies $T_{\mathfrak{n}} T \mathcal{M}$ with the direct sum $T_{p} \mathcal{M} \oplus T_{p} \mathcal{M}$ using the 
horizontal space determined by the Levi-Civita connection then $T_{\mathfrak{n}}\left(T \mathcal{P}^{\perp}\right)$ is given by (compare with (2.2)):

$$
T_{\mathfrak{n}}\left(T \mathcal{P}^{\perp}\right)=\left\{(v, w) \in T_{p} \mathcal{M} \oplus T_{p} \mathcal{M}: v \in T_{p} \mathcal{P}, w+A_{\mathfrak{n}}(v) \in T_{p} \mathcal{P}^{\perp}\right\} .
$$

In the corollary below we denote by $G_{n}(T \mathcal{M})$ the fiber bundle over $T \mathcal{M}$ whose fiber at $v \in T \mathcal{M}$ is the Grassmannian of all $n$-dimensional subspaces of the tangent space $T_{v} T \mathcal{M}$. Observe that $T_{\mathfrak{n}}\left(T \mathcal{P}^{\perp}\right)$ is then a point in $G_{n}(T \mathcal{M})$.

Corollary 4.7. Let $(\mathcal{M}, \mathfrak{g})$ be an n-dimensional semi-Riemannian manifold. Assume that we are given a family of pairs $\left(\mathcal{P}_{j}, \gamma_{j}\right), j \in \mathbb{N} \cup\{\infty\}$, where each $\mathcal{P}_{j}$ is a submanifold of $\mathcal{M}$ and each $\gamma_{j}:[a, b] \rightarrow \mathcal{M}$ is a geodesic starting orthogonally at $\mathcal{P}_{j}$. Assume that:

(1) $\mathcal{P}_{j}$ is nondegenerate at $\gamma_{j}(a)$ for all $j \in \mathbb{N} \cup\{\infty\}$;

(2) $\operatorname{dim}\left(\mathcal{P}_{j}\right)=\operatorname{dim}\left(\mathcal{P}_{\infty}\right)$ for all $j \in \mathbb{N}$;

(3) all $\mathcal{P}_{j}$-focal points along $\gamma_{j}$ are nondegenerate, for all $j \in \mathbb{N} \cup\{\infty\}$;

(4) $\gamma_{\infty}(b)$ is not $\mathcal{P}_{\infty}$-focal along $\gamma_{\infty}$;

(5) $\gamma_{j}^{\prime}\left(\right.$ a) $\rightarrow \gamma_{\infty}^{\prime}($ a) in $T \mathcal{M}$ as $j \rightarrow \infty$;

(6) $T_{\gamma_{j}^{\prime}(a)}\left(T \mathcal{P}_{j}^{\perp}\right) \rightarrow T_{\gamma_{\infty}^{\prime}(a)}\left(T \mathcal{P}_{\infty}^{\perp}\right)$ in $G_{n}(T \mathcal{M})$ as $j \rightarrow \infty$.

Then, for $j$ large enough, $\gamma_{j}(b)$ is not $\mathcal{P}_{j}$-focal along $\gamma_{j}$ and $\mathrm{i}_{\mathrm{foc}}\left(\gamma_{j}, \mathcal{P}_{j}\right)=$ $\mathrm{i}_{\text {foc }}\left(\gamma_{\infty}, \mathcal{P}_{\infty}\right)$.

Proof. Choose a smooth referential around $\gamma_{\infty}(a)$ and parallel transport such referential along each $\gamma_{j}$. The conclusion follows by applying Theorem 4.6 to the quadruples $\left(g_{j}, R_{j}, P_{j}, S_{j}\right)$ obtained from $\left(\mathcal{M}, \mathfrak{g}, \mathcal{P}_{j}, \gamma_{j}\right)$.

Corollary 4.8. Under the hypothesis of Corollary 4.7, if $(\mathcal{M}, \mathfrak{g})$ is Riemannian or if $(\mathcal{M}, \mathfrak{g})$ is Lorentzian and $\gamma_{j}$ is nonspacelike for all $j \in$ $\mathbb{N} \cup\{\infty\}$, then for $j$ sufficiently large, the sum of the multiplicities of the $\mathcal{P}_{j}$ focal points along $\gamma_{j}$ is equal to the sum of the multiplicities of the $\mathcal{P}_{\infty}$-focal points along $\gamma_{\infty}$.

Hypothesis (2) is essential for Corollary 4.7 as the following example shows.

Example 4.9. Consider the plane $\mathcal{M}=\mathbb{R}^{2}$ endowed with the standard Euclidean metric $\mathfrak{g}$. For $j \in \mathbb{N}$, let $\mathcal{P}_{j} \subset \mathbb{R}^{2}$ be the circle centered at $\left(0, \frac{1}{j}\right)$ and passing through the origin; then the segment $[0,1] \ni t \mapsto \gamma(t)=(0, t)$ is a geodesic starting orthogonally to $\mathcal{P}_{j}$ and containing a unique $\mathcal{P}_{j}$-focal point at $\left(0, \frac{1}{j}\right)$ whose multiplicity (and signature) is equal to 1 . Hence $\mathrm{i}_{\text {foc }}\left(\gamma, \mathcal{P}_{j}\right)=1$. We have $T_{\gamma^{\prime}(0)}\left(T \mathcal{P}_{j}^{\perp}\right)=\operatorname{span}\left\{\left(\frac{1}{j}, 0,-1,0\right),(0,0,0,1)\right\}$; thus $T_{\gamma^{\prime}(0)}\left(T \mathcal{P}_{j}^{\perp}\right) \rightarrow\{0\} \times \mathbb{R}^{2}=T_{\gamma^{\prime}(0)}\left(T \mathcal{P}_{\infty}^{\perp}\right)$, where $\mathcal{P}_{\infty}=\{0\}$. But $\gamma$ has no $\mathcal{P}_{\infty}$-focal (i.e., conjugate) points and thus $\mathrm{i}_{\text {foc }}\left(\gamma, \mathcal{P}_{\infty}\right)=0$. 
Lemma 4.10. Let $\mathcal{X}$ be a topological space. For each $\lambda \in \mathcal{X}$, let $\left(g_{\lambda}, R_{\lambda}\right.$, $\left.P_{\lambda}, S_{\lambda}\right)$ be an admissible quadruple for the differential problem in $\mathbb{R}^{n}$. Assume that $\operatorname{dim}\left(P_{\lambda}\right)=r$ for all $\lambda \in \mathcal{X}$ and assume that the maps:

$$
\begin{gathered}
\mathcal{X} \ni \lambda \longmapsto g_{\lambda} \in \operatorname{Bil}_{\text {sym }}\left(\mathbb{R}^{n}\right), \quad \mathcal{X} \times[a, b] \ni(\lambda, t) \longmapsto R_{\lambda}(t) \in \operatorname{Lin}\left(\mathbb{R}^{n}\right), \\
\mathcal{X} \ni \lambda \longmapsto\left(P_{\lambda}, S_{\lambda}\right) \in \bigvee_{2} E_{r, n}^{*},
\end{gathered}
$$

are continuous. Then, given $\lambda_{0} \in \mathcal{X}$, there exists $\varepsilon>0$ and a neighborhood $\mathcal{V}$ of $\lambda_{0}$ in $\mathcal{X}$ such that $\left(g_{\lambda}, R_{\lambda}, P_{\lambda}, S_{\lambda}\right)$ has no $\left(P_{\lambda}, S_{\lambda}\right)$-focal instants in ]$a, a+\varepsilon]$ for all $\lambda \in \mathcal{V}$.

Proof. Arguing as in the proof of Proposition 2.2, we can find continuous maps $(t, \lambda) \mapsto \widetilde{J}_{i}(t, \lambda), i=1, \ldots, n$, such that $\left(\widetilde{J}_{i}\left(a, \lambda_{0}\right)\right)_{i=1}^{n}$ is a basis of $\mathbb{R}^{n}$ and such that $\left.\left.t \in\right] a, b\right]$ is $\left(P_{\lambda}, S_{\lambda}\right)$-focal for $\left(g_{\lambda}, R_{\lambda}, P_{\lambda}, S_{\lambda}\right)$ iff $\operatorname{det}\left(\widetilde{J}_{i}(t, \lambda)\right)_{i=1}^{n}=0$. The conclusion follows.

\section{Curves of Lagrangians originating from differential problems.}

Given an admissible quadruple for the differentiable problem $(g, R, P, S)$, one can construct objects $\Phi, \ell_{0}$ and $\ell$ as in Subsection 4.1. In Subsections 5.1, 5.2 and 5.3 we investigate the inverse problem: When does a curve $\ell:[a, b] \rightarrow \Lambda$ arises from a quadruple $(g, R, P, S)$ ? In Subsection 5.4 we use the theory developed in the earlier subsections to provide a counterexample to the equality between the Maslov and the focal index of a quadruple $(g, R, P, S)$ having degenerate focal instants. More specifically, we give an example of an admissible quadruple $(g, R, P, S)$ in $\mathbb{R}^{2}$, with $R$ real-analytic, $n_{+}(g)=1$, $P=\{0\}$, having a unique $(P, S)$-focal instant, and whose Maslov index is equal to -1 , while the focal index is equal to zero.

It is proven in [14, Section 3] that every admissible quadruple for the differential problem in $\mathbb{R}^{n}$ with $P=\{0\}$ and $R$ smooth can be obtained from an admissible quadruple for the geometric problem $(\mathcal{M}, \mathfrak{g}, \gamma, \mathcal{P})$, with $\mathcal{P}=\{\gamma(a)\}, \operatorname{dim}(\mathcal{M})=n+1$, by a parallel trivialization of the normal bundle along $\gamma$. Moreover, $(\mathcal{M}, \mathfrak{g})$ can be chosen to be conformally flat and $\gamma$ can be chosen to be either timelike or spacelike. In particular, the counterexample given in Subsection 5.4 actually admits a geometric realization in terms of a spacelike geodesic $\gamma$ on a real-analytic conformally flat three-dimensional Lorentzian manifold $(\mathcal{M}, \mathfrak{g})$.

\subsection{Differential problems generate curves of Lagrangians tangent} to affine distributions. Let $g \in \operatorname{Bil}_{\text {sym }}\left(\mathbb{R}^{n}\right)$ be a fixed nondegenerate symmetric bilinear form in $\mathbb{R}^{n}$. Let $\widehat{\mathcal{D}}$ denote the right-invariant distribution of affine spaces in the Lie group $\operatorname{Sp}(2 n, \mathbb{R})$ whose value at the identity is the affine subspace of the Lie algebra $\operatorname{sp}(2 n, \mathbb{R})$ consisting of those linear endomorphisms of $\mathbb{R}^{n} \oplus \mathbb{R}^{n *}$ that can be written in block-matrix form as $X=\left(\begin{array}{ll}0 & g^{-1} \\ C & 0\end{array}\right)$, with $C \in \operatorname{Bil}_{\text {sym }}\left(\mathbb{R}^{n}\right)$ symmetric. From (4.2) and (4.4) 
it follows directly that a $C^{1}$ curve $\Phi:[a, b] \rightarrow \operatorname{Sp}(2 n, \mathbb{R})$ with $\Phi(a)=\mathrm{Id}$ arises from an admissible quadruple for the differential problem $(g, R, P, S)$ if and only if $\Phi$ is tangent to the distribution $\widehat{\mathcal{D}}$, i.e., if $\Phi^{\prime}(t) \in \widehat{\mathcal{D}}_{\Phi(t)}$ for all $t \in[a, b]$.

Now let $\ell_{0} \in \Lambda$ be fixed and consider the fibration $\beta_{\ell_{0}}: \operatorname{Sp}(2 n, \mathbb{R}) \rightarrow$ $\Lambda$ given by evaluation at $\ell_{0}$. The curve of Lagrangians $\ell$ arising from $(g, R, P, S)$ is simply the projection on $\Lambda$ of the curve $\Phi$ (recall (4.9)). Since the Lie group $\operatorname{Sp}(2 n, \mathbb{R})$ acts on $\Lambda$ on the left, the right-invariant distribution $\widehat{\mathcal{D}}$ on $\operatorname{Sp}(2 n, \mathbb{R})$ is projectable on $\Lambda$ and such projection does not depend on the choice of $\ell_{0}$. More explicitly, we define a distribution of affine spaces $\mathcal{D}$ on $\Lambda$ by setting $\mathcal{D}_{L}=\mathrm{d} \beta_{L}(\mathrm{Id})\left(\widehat{\mathcal{D}}_{\text {Id }}\right) \subset T_{L} \Lambda$, for all $L \in \Lambda$. It is easy to see that if $\Phi:[a, b] \rightarrow \operatorname{Sp}(2 n, \mathbb{R})$ is tangent to $\widehat{\mathcal{D}}$ and if $\ell=\beta_{\ell_{0}} \circ \Phi$ for some $\ell_{0}$ then $\ell$ is tangent to $\mathcal{D}$. Hence, tangency to $\mathcal{D}$ is a necessary condition for a $C^{1}$ curve $\ell$ on $\Lambda$ to arise from some quadruple $(g, R, P, S)$. We remark that the distribution $\mathcal{D}$ on $\Lambda$ does not have constant rank. Using Formula (3.2) for $\mathrm{d} \beta_{L}$ one computes easily:

$$
\mathcal{D}_{L}=\left\{\left.\left(0 \oplus g^{-1}\right)\right|_{L}+B: B \in \operatorname{Bil}_{\text {sym }}(L), L_{0} \cap L \subset \operatorname{Ker}(B)\right\},
$$

where $L_{0}=\{0\} \oplus \mathbb{R}^{n *}$ and $0 \oplus g^{-1} \in \operatorname{Bil}_{\text {sym }}\left(\mathbb{R}^{n} \oplus \mathbb{R}^{n *}\right)$ denotes the symmetric bilinear form $\left(0 \oplus g^{-1}\right)\left(\left(v_{1}, \alpha_{1}\right),\left(v_{2}, \alpha_{2}\right)\right)=g^{-1}\left(\alpha_{1}, \alpha_{2}\right)$. Thus, for $L \in$ $\Lambda_{k}\left(L_{0}\right)$, we have $\operatorname{dim}\left(\mathcal{D}_{L}\right)=\frac{1}{2}(n-k)(n-k+1)$. In particular $\mathcal{D}$ has constant rank on each manifold $\Lambda_{k}\left(L_{0}\right)$; in the open dense set $\Lambda_{0}\left(L_{0}\right)$, the distribution $\mathcal{D}$ is equal to the entire tangent bundle of $\Lambda$. Hence, the condition $\ell^{\prime} \in \mathcal{D}$ is vacuous outside the variety $\Lambda_{\geq 1}\left(L_{0}\right)$, but it gives some information on how $\ell$ should intersect $\Lambda_{\geq 1}\left(L_{0}\right)$.

\subsection{A study of curves of Lagrangians in local coordinates. Let $L_{1} \subset$} $\mathbb{R}^{n} \oplus \mathbb{R}^{n *}$ be a Lagrangian complementary to $L_{0}=\{0\} \oplus \mathbb{R}^{n *}$. We study the curve $\ell:[a, b] \rightarrow \Lambda$ arising from a quadruple $(g, R, P, S)$ in the chart $\phi_{L_{0}, L_{1}}$. The Lagrangian $L_{1}$ can be written in the form:

$$
L_{1}=\left\{(v, Z v): v \in \mathbb{R}^{n}\right\},
$$

where $Z: \mathbb{R}^{n} \rightarrow \mathbb{R}^{n *}$ is symmetric. We set $\beta(t)=\phi_{L_{0}, L_{1}}(\ell(t)) \in \operatorname{Bil}_{\text {sym }}\left(L_{0}\right)$, for $t$ in some subinterval of $[a, b]$. We identify $\beta(t)$ with a symmetric bilinear form on $\mathbb{R}^{n *}$ and with a linear map $\beta(t): \mathbb{R}^{n *} \rightarrow \mathbb{R}^{n}$. Then $\ell(t)$ is the graph of $L_{0} \ni(0, \alpha) \longmapsto(\beta(t) \alpha, Z \beta(t) \alpha) \in L_{1}$. Obviously $\beta^{\prime}(t)=\mathrm{d} \phi_{L_{0}, L_{1}}(\ell(t))$. $\ell^{\prime}(t)$; from (4.10) and (3.3) we get:

$$
\beta^{\prime}(t)=\omega(X(t) \eta(t) \cdot, \eta(t) \cdot),
$$

where $\eta(t): L_{0} \rightarrow \ell(t)$ is the isomorphism given by the restriction to $L_{0}$ of the projection onto $\ell(t)$ corresponding to the decomposition $\mathbb{R}^{n} \oplus \mathbb{R}^{n *}=$ $\ell(t) \oplus L_{1}$. One computes:

$$
\eta(t): L_{0} \ni(0, \alpha) \longmapsto(\beta(t) \alpha, \alpha+Z \beta(t) \alpha) \in \ell(t) .
$$


From (4.2), (5.2) and (5.3) we get:

$$
\beta^{\prime}(t)=g^{-1}+\beta(t) Z g^{-1}+g^{-1} Z \beta(t)+\beta(t)\left(Z g^{-1} Z-g R(t)\right) \beta(t) .
$$

We call (5.4) the generalized Riccati equation.

Our goal is, given a fixed nondegenerate symmetric bilinear form $g \in$ $\operatorname{Bil}_{\text {sym }}\left(\mathbb{R}^{n}\right)$, to determine conditions for a curve $\beta$ on $\operatorname{Bil}_{\text {sym }}\left(\mathbb{R}^{n *}\right)$ to be the solution of (5.4) for some curve $R:[a, b] \rightarrow \operatorname{Lin}\left(\mathbb{R}^{n}\right)$ of $g$-symmetric endomorphisms of $\mathbb{R}^{n}$. Observe that if $\beta(t)$ is invertible for some $t$ then $R(t)$ must be given by:

$$
R(t)=g^{-1}\left[\beta(t)^{-1}\left(g^{-1}+\beta(t) Z g^{-1}+g^{-1} Z \beta(t)-\beta^{\prime}(t)\right) \beta(t)^{-1}+Z g^{-1} Z\right]
$$

obviously the right-hand side of (5.5) is $g$-symmetric, so that we indeed get a curve $R$ of $g$-symmetric endomorphisms of $\mathbb{R}^{n}$ in every interval on which $\beta$ is invertible. Observe that this is consistent with the results of Subsection 5.1. We now study the derivatives of $\beta$ at those instants $t$ where $\beta(t)$ is degenerate. First, observe that (5.4) implies that:

$$
\begin{aligned}
\beta^{\prime}(t)\left(\alpha_{1}, \alpha_{2}\right)=\left(g^{-1}+\beta(t) Z g^{-1}+g^{-1} Z \beta(t)\right)\left(\alpha_{1}, \alpha_{2}\right) & \\
& \text { if } \alpha_{1} \in \operatorname{Ker}(\beta(t)) \text { or if } \alpha_{2} \in \operatorname{Ker}(\beta(t)) .
\end{aligned}
$$

It is easy to see that (5.6) is just the coordinate version of the condition that $\ell$ be tangent to $\mathcal{D}$. By taking another derivative of (5.4), we obtain:

$$
\beta^{\prime \prime}(t)\left(\alpha_{1}, \alpha_{2}\right)=\left(\beta^{\prime}(t) Z g^{-1}+g^{-1} Z \beta^{\prime}(t)\right)\left(\alpha_{1}, \alpha_{2}\right), \quad \alpha_{1}, \alpha_{2} \in \operatorname{Ker}(\beta(t)) ;
$$

finally, from (5.6) and (5.7) we obtain the following second order condition for $\beta$ :

$$
\begin{array}{r}
\beta^{\prime \prime}(t)\left(\alpha_{1}, \alpha_{2}\right)=2\left(g^{-1} Z g^{-1}+g^{-1} Z \beta(t) Z g^{-1}\right)\left(\alpha_{1}, \alpha_{2}\right) \\
\alpha_{1}, \alpha_{2} \in \operatorname{Ker}(\beta(t)) .
\end{array}
$$

In Subsection 5.3 below we will show that conditions (5.6) and (5.8) are sufficient for $\beta$ to arise from some $R$ in a nondegenerate case.

\subsection{The case where $\boldsymbol{\beta}^{\prime}(t)$ is nondegenerate on $\operatorname{Ker}(\boldsymbol{\beta}(t))$. Consider} a smooth curve $\beta:[a, b] \rightarrow \operatorname{Bil}_{\text {sym }}\left(\mathbb{R}^{n *}\right)$. Assume that, for every $t \in[a, b]$ for which $\beta(t)$ is degenerate, the derivative $\beta^{\prime}(t)$ is nondegenerate on $\operatorname{Ker}(\beta(t))$ and conditions (5.6) and (5.8) hold. We will prove that there exists a unique smooth curve $R:[a, b] \rightarrow \operatorname{Lin}\left(\mathbb{R}^{n}\right)$ of $g$-symmetric endomorphisms of $\mathbb{R}^{n}$ such that (5.4) holds. Then, if $P \subset \mathbb{R}^{n}$ is a nondegenerate subspace for $g$, $S \in \operatorname{Bil}_{\text {sym }}(P)$ is a symmetric bilinear form, $\ell_{0}$ is defined as in (4.5) and $\beta(a)=\phi_{L_{0}, L_{1}}(\ell(a))$, it follows that the curve of Lagrangians $\ell=\phi_{L_{0}, L_{1}}^{-1} \circ \beta$ arises from $(g, R, P, S)$, where $L_{1}$ is defined in (5.1). 
In order to achieve our goal, we start by observing that Lemma 3.7 implies that there are only a finite number of instants $t \in[a, b]$ with $\beta(t)$ degenerate. Formula (5.5) can be used to define $R$ at those $t$ where $\beta(t)$ is invertible. We now fix $t_{0} \in[a, b]$ with $\beta\left(t_{0}\right)$ degenerate and we show that $R$ admits a smooth extension to $t_{0}$.

Let $\left(b_{i}\right)_{i=1}^{n}$ be a basis of $\mathbb{R}^{n *}$ such that $\left(b_{i}\right)_{i=1}^{k}$ is a basis of $\operatorname{Ker}\left(\beta\left(t_{0}\right)\right)$; the restriction of $\beta\left(t_{0}\right)$ to the span of $\left(b_{i}\right)_{i=k+1}^{n}$ is clearly nondegenerate. We will think of the bilinear forms on $\mathbb{R}^{n *}$ as matrices relative to the basis $\left(b_{i}\right)_{i=1}^{n}$. Observe that $\beta\left(t_{0}\right)$ has zeroes outside the lower-right $(n-k) \times(n-k)$ block.

For $t \neq t_{0}$, let $\widetilde{\beta}(t)$ be the matrix obtained by dividing the first $k$ columns of $\beta(t)$ by $t-t_{0}$; we define $\widetilde{\beta}\left(t_{0}\right)$ by replacing the first $k$ columns of $\beta\left(t_{0}\right)$ with the first $k$ columns of $\beta^{\prime}\left(t_{0}\right)$. It is easy to see that $t \mapsto \widetilde{\beta}(t)$ is smooth around $t_{0}$. For $a \in \mathbb{R}$, we set $D_{k}(a)=\left(\begin{array}{cc}a \mathrm{I}_{k} & 0 \\ 0 & \mathrm{I}_{n-k}\end{array}\right)$, where $\mathrm{I}_{j}$ denotes the $j \times j$ identity matrix. Then $\beta(t)=\widetilde{\beta}(t) D_{k}\left(t-t_{0}\right)=D_{k}\left(t-t_{0}\right) \widetilde{\beta}(t)^{*}$, for all $t \neq t_{0}$. Hence:

$$
\beta(t)^{-1}=D_{k}\left(\frac{1}{t-t_{0}}\right) \widetilde{\beta}(t)^{-1}=\left(\widetilde{\beta}(t)^{-1}\right)^{*} D_{k}\left(\frac{1}{t-t_{0}}\right),
$$

for all $t \neq t_{0}$. Since $\beta^{\prime}\left(t_{0}\right)$ is nondegenerate on $\operatorname{Ker}\left(\beta\left(t_{0}\right)\right)$, the matrix $\widetilde{\beta}\left(t_{0}\right)$ is invertible and so $t \mapsto \widetilde{\beta}(t)^{-1}$ is smooth around $t_{0}$. From (5.5), in order to prove that $R$ has a smooth extension to $t_{0}$, it suffices to show that the map $t \mapsto \beta(t)^{-1} Q(t) \beta(t)^{-1}$ has a smooth extension to $t_{0}$, where $Q$ is defined by:

$$
Q(t)=g^{-1}+\beta(t) Z g^{-1}+g^{-1} Z \beta(t)-\beta^{\prime}(t) .
$$

We claim that $Q\left(t_{0}\right)$ is zero outside the lower-right $(n-k) \times(n-k)$-block and that $Q^{\prime}\left(t_{0}\right)$ is zero on the upper-left $k \times k$-block. Namely, it is easy to check that (5.6) and (5.8) imply (5.7). Then (5.7) and (5.10) imply that $Q\left(t_{0}\right)\left(\alpha_{1}, \alpha_{2}\right)=0$ if either $\alpha_{1}$ or $\alpha_{2}$ is in $\operatorname{Ker}\left(\beta\left(t_{0}\right)\right)$ and that $Q^{\prime}\left(t_{0}\right)\left(\alpha_{1}, \alpha_{2}\right)=$ 0 if both $\alpha_{1}$ and $\alpha_{2}$ are in $\operatorname{Ker}\left(\beta\left(t_{0}\right)\right)$. This proves the claim. We can now write:

$$
Q(t)=D_{k}\left(t-t_{0}\right) \widetilde{Q}(t) D_{k}\left(t-t_{0}\right),
$$

with $t \mapsto \widetilde{Q}(t)$ smooth around $t_{0}$. From (5.9), (5.11) and the fact that $t \mapsto \widetilde{\beta}(t)^{-1}$ is smooth around $t_{0}$, it follows that $t \mapsto \beta(t)^{-1} Q(t) \beta(t)^{-1}$ (and thus $t \mapsto R(t))$ is smooth around $t_{0}$.

5.4. A counterexample to the equality $\mathrm{i}_{\text {Maslov }}(g, R, P, S)=\mathrm{i}_{\text {foc }}(g, R$, $\boldsymbol{P}, \boldsymbol{S})$. In Subsection 5.3 we have shown that, if $\beta^{\prime}(t)$ is nondegenerate on $\operatorname{Ker}(\beta(t))$, then conditions (5.6) and (5.8) are sufficient for the existence of a smooth map $R$ for which $\beta$ is a solution of (5.4). It will become clear below that the hypothesis that $\beta^{\prime}(t)$ be nondegenerate on $\operatorname{Ker}(\beta(t))$ is indeed essential here; namely, if $\beta^{\prime}\left(t_{0}\right)$ is degenerate on $\operatorname{Ker}\left(\beta\left(t_{0}\right)\right)$ for some $t_{0}$ then one has to impose conditions on the higher order derivatives of $\beta$ at $t_{0}$ in 
order to guarantee that the map $t \mapsto R(t)$ defined by (5.5) has a smooth extension to $t_{0}$.

We consider the following setup. Set $n=2$ and define the objects $g$, $Z$ and $P$ by $g=\left(\begin{array}{ll}0 & 1 \\ 1 & 0\end{array}\right), Z=\left(\begin{array}{ll}0 & 0 \\ 0 & 1\end{array}\right), P=\{0\}$. We will describe a curve $\beta:[a, b] \rightarrow \operatorname{Bil}_{\mathrm{sym}}\left(\mathbb{R}^{2^{*}}\right)$,

$$
\beta(t)=\left(\begin{array}{ll}
x(t) & z(t) \\
z(t) & y(t)
\end{array}\right)
$$

where $x, y$ and $z$ are real-analytic scalar functions on $[a, b], a<0<b$, such that the following properties are satisfied:

(1) $x(a)=y(a)=z(a)=0$;

(2) $x^{\prime}(a)=y^{\prime}(a)=0, z^{\prime}(a)=1, x^{\prime \prime}(a)=2$ and $y^{\prime \prime}(a)=z^{\prime \prime}(a)=0$;

(3) $x(t) y(t)-z(t)^{2}$ vanishes precisely for $t=a$ and for $t=0$;

(4) $x(0)=z(0)=0$ and $y(0)=-1$;

(5) $x^{\prime}(0)=x^{\prime \prime}(0)=0$ and $z^{\prime}(0)=0$;

(6) $x^{\prime \prime \prime}(0)<0$;

(7) the map $t \mapsto R(t)$ defined by (5.5) has an extension to $t=0$ which is real-analytic around $t=0$.

Once a curve $\beta$ satisfying properties (1)-(7) above is determined, the desired counterexample can be easily constructed. Namely, define $R$ by (5.5). From condition (3), $R$ is real-analytic on $[a, b] \backslash\{a, 0\}$; condition (7) says that $R$ has a real-analytic extension to 0 . A simple computation shows that, under condition (1), condition (2) is equivalent to (5.6) and (5.8) at $t=a$. Thus, the result proved in Subsection 5.3 guarantees that $R$ has a real-analytic extension to $t=a$. Now define $L_{1}$ by (5.1), $L_{0}$ by (4.6) and set $\ell=\phi_{L_{0}, L_{1}}^{-1} \circ \beta$. Condition (1) implies that $\ell(a)=L_{0}$ and therefore $\ell$ is the curve of Lagrangians corresponding to the quadruple $(g, R, P, S)$. By condition (3), $t=0$ is the unique $(P, S)$-focal instant; condition (4) implies that the kernel of $\beta(0)$ is spanned by the first vector of the canonical basis of $\mathbb{R}^{2 *}$ and thus the signature of the $(P, S)$-focal instant $t=0$ is zero (recall (3.4) and (4.8)). Hence $\mathrm{i}_{\text {foc }}(g, R, P, S)=0$. On the other hand:

$$
\mathrm{i}_{\text {Maslov }}(g, R, P, S)=-1 \text {; }
$$

namely, recall from Proposition 3.9 that $\mathrm{i}_{\text {Maslov }}(g, R, P, S)=\mu_{L_{0}}\left(\left.\ell\right|_{[a+\varepsilon, b]}\right)$ equals the variation of the coindex of $\beta(t)$ near $t=0$. Conditions (4), (5) and (6) imply $n_{+}(\beta(t))=1$ for $t<0$ near zero and $n_{+}(\beta(t))=0$ for $t>0$ near zero; this yields (5.13).

We will now show that it is possible to determine polynomial functions $x$, $y$ and $z$ satisfying conditions (1)-(7). We start by analyzing condition (7). As in Subsection 5.3, $t \mapsto R(t)$ has a real-analytic extension to $t=0$ if and only if $t \mapsto \beta(t)^{-1} Q(t) \beta^{-1} Q(t)$ does, where $Q$ is given by (5.10). Consider 
the matrix $\hat{\beta}(t)=\left(\begin{array}{cc}y(t) & -z(t) \\ -z(t) & x(t)\end{array}\right)$, so that

$$
\beta(t)^{-1} Q(t) \beta(t)^{-1}=\frac{1}{\operatorname{det}(\beta(t))^{2}} \hat{\beta}(t) Q(t) \hat{\beta}(t),
$$

when $\beta(t)$ is invertible. We have:

$$
\hat{\beta}(t) Q(t) \hat{\beta}(t)=\left(\begin{array}{ll}
p_{1}(t) & p_{2}(t) \\
p_{2}(t) & p_{3}(t)
\end{array}\right),
$$

where $p_{1}=-x^{\prime} y^{2}-2 y z+2 y z z^{\prime}-y^{\prime} z^{2}, p_{2}=x^{\prime} y z-y z^{2}+x y-x y z^{\prime}+x y^{2}+$ $z^{2}-z^{2} z^{\prime}+x y^{\prime} z$ and $p_{3}=-x^{\prime} z^{2}+2 z^{3}-2 x z+2 x z z^{\prime}-2 x y z-x^{2} y^{\prime}$. Conditions (4), (5) and (6) imply that $t \mapsto \operatorname{det}(\beta(t))$ has a zero of order 3 at $t=0$; thus condition (7) is equivalent to the maps $p_{i}(t), i=1,2,3$, having zeroes of order at least 6 at $t=0$. This can be achieved, for instance, by setting:

$$
x(t)=-2 t^{3}-\frac{54}{5} t^{5}, \quad y(t)=-1-6 t+18 t^{2}-54 t^{3}, \quad z(t)=-3 t^{2},
$$

for $t$ near zero. Observe that the polynomials above also satisfy conditions (4)-(6). For $t$ near $a$, we take $x, y, z$ to be arbitrary smooth maps satisfying (1) and (2). It is easy to see that $n_{+}(\beta(t))=1$ for $t>a$ near $a$ and for $t<0$ near zero. Since the set of invertible symmetric matrices of coindex 1 is connected and open in $\operatorname{Bil}_{\text {sym }}\left(\mathbb{R}^{2^{*}}\right)$, we may then extend $x, y, z$ smoothly to $[a, 0]$ in such a way that $\beta(t)$ is invertible for all $t \in] a, 0[$; we then extend $x, y, z$ smoothly to a slightly larger interval $[a, b], b>0$, so that condition (3) is satisfied.

The construction above yields the desired counterexample in the smooth case. We now use a density argument to show the existence of a real-analytic counterexample.

Let $x, y$ and $z$ be given smooth functions such that conditions (1)-(7) are satisfied; we start by observing that if $\widetilde{x}, \widetilde{y}$ and $\widetilde{z}$ are smooth functions having the first six derivatives at $t=0$ and the first two derivatives at $t=a$ equal to the corresponding derivatives of $x, y$ and $z$, then, by replacing $x, y$ and $z$ with $\widetilde{x}, \widetilde{y}$ and $\widetilde{z}$, only condition (3) may fail to hold. If such a replacement is made in such a way that $\widetilde{x}, \widetilde{y}$ and $\widetilde{z}$ are sufficiently close to $x, y$ and $z$ in the $C^{4}$-topology, then also condition (3) will remain true. To prove this, we apply the following elementary lemma to the function $f(t)=x(t) y(t)-z(t)^{2}$ on the interval $[a, 0]$ with $k=3$ :

Lemma 5.1. Let $k \in \mathbb{N}$ and let $f:[c, d] \rightarrow \mathbb{R}$ be a function of class $C^{k+1}$. Assume that $f$ has zeroes precisely at the endpoints $c, d$ and that these zeroes are of order at the most $k$. Then, there exists a neighborhood $\mathcal{U}$ of $f$ in the $C^{k+1}$-topology such that, every $g \in \mathcal{U}$ having the same order of zeroes as $f$ at $c$ and $d$ has no zeroes in $] c, d[$. 
The proof of the existence of polynomial functions $x, y, z:[a, b] \rightarrow \mathbb{R}$ satisfying conditions (1)-(7) now follows by applying the elementary density lemma below in the interval $[a, 0]$, with $k=6$.

Lemma 5.2. Let $k \in \mathbb{N}$ and $p_{i}, q_{i} \in \mathbb{R}, i=0, \ldots, k$, be fixed. Let $A$ denote the affine subspace of $C^{k}([a, b], \mathbb{R})$ consisting of maps $f$ with $f^{(i)}(c)=p_{i}$, $f^{(i)}(d)=q_{i}, i=0, \ldots, k$. Then the subset of $A$ consisting of polynomials maps is dense in $A$ with respect to the $C^{k}$-topology.

Acknowledgements. The authors wish to express their gratitude to Professor Daniel Baumann Henry, Professor Daciberg Lima Gonçalves and Professor Volker Perlick who have given many fruitful suggestions.

\section{References}

[1] V.I. Arnol'd, Characteristic class entering in quantization conditions, Funct. Anal. Appl., 1 (1967), 1-13, MR 35 \#2296, Zbl 0175.20303.

[2] J. Beem and P. Ehrlich, A Morse index theorem for null geodesics, Duke Math. J., 46 (1979), 561-569, MR 80k:53097, Zbl 0418.58008.

[3] J.K. Beem, P.E. Ehrlich and K.L. Easley, Global Lorentzian Geometry, Marcel Dekker, Inc., New York and Basel, 1996, MR 97f:53100, Zbl 0846.53001.

[4] R. Bott, On the iteration of closed geodesics and the Sturm intersection theory, Commun. Pure Appl. Math., 9 (1956), 171-206, MR 19,859f, Zbl 0074.17202.

[5] E.A. Coddington and N. Levinson, Theory of Ordinary Differential Equations, McGraw Hill Book Company, New York, Toronto, London, 1955, MR 16,1022b, Zbl 0064.33002.

[6] J.C. Corrêa Eidam, A.L. Pereira, P. Piccione and D.V. Tausk, On the equality between the Maslov index and the spectral index for the semi-Riemannian Jacobi operator, J. Math. Anal. Appl., 268(2) (2002), 564-589, CMP 1896215.

[7] M. do Carmo, Riemannian Geometry, Birkhäuser, Boston, 1992, MR 92i:53001, Zbl 0752.53001.

[8] H.M. Edwards, A generalized Sturm theorem, Ann. of Math., 80 (1964), 22-57, MR 29 \#1652, Zbl 0127.03805.

[9] P.E. Ehrlich and S. Kim, A focal index theorem for null geodesics, J. Geom. Phys., 6(4) (1989), 657-670, MR 91m:53050, Zbl 0709.53044.

[10] D.B. Fuks, Maslov-Arnol'd characteristic classes, Soviet Math. Dokl., 9(1) (1968), 96-99, MR 37 \#934, Zbl 0175.20304.

[11] F. Giannoni, A. Masiello and P. Piccione, A Morse theory for massive particles and photons by Fermat principles in general relativity, J. Geom. Phys., 35(1) (2000), 1-35, MR 2001h:58012, Zbl 0965.83017.

[12] F. Giannoni, A. Masiello, P. Piccione and D. Tausk, A generalized index theorem for Morse-Sturm systems and applications to semi-Riemannian geometry, Asian J. Math., 5(3) (2001), 441-472, CMP 1868574.

[13] V. Guillemin and S. Sternberg, Geometric Asymptotics, Mathematical Surveys and Monographs, 14, AMS, Providence RI, 1990, MR 58 \#24404, Zbl 0364.53011. 
[14] A.D. Helfer, Conjugate points on spacelike geodesics or pseudo-self-adjoint MorseSturm-Liouville systems, Pacific J. Math., 164(2) (1994), 321-340, MR 95j:58026, Zbl 0799.58018.

[15] B. O'Neill, Semi-Riemannian Geometry with Applications to Relativity, Academic Press, New York, 1983, MR 85f:53002, Zbl 0531.53051.

[16] P. Piccione and D.V. Tausk, A note on the Morse index theorem for geodesics between submanifolds in semi-Riemannian geometry, J. Math. Phys., 40(12) (1999), 66826688, MR 2000k:58018, Zbl 0982.53037.

[17] _ An index theorem for non-periodic solutions of Hamiltonian systems, Proc. London Math. Soc., 83 (2001), 351-389, MR 2002f:53135.

[18] F. Treves, Introduction to Pseudodifferential Operators, Plenum, New York, 1982, MR 82i:58068, Zbl 0547.47031.

[19] V.S. Varadarajan, Lie Groups, Lie Algebras and Their Representations, Prentice-Hall series in Modern Analysis, 1974, New Jersey, MR 51 \#13113, Zbl 0371.22001.

Received January 5, 2000 and revised April 15, 2002. The authors are partially sponsored by $\mathrm{CNPq}$ (Brazil).

Departamento de Matemática

Universidade Estadual DE CAMPINAS

SP, BRAZIL

E-mail address: mercuri@ime.unicamp.br

Departamento de Matemática

Universidade DE SÃo PAUlo

BRAZIL

E-mail address: piccione@ime.usp.br

Departamento de Matemática

Universidade DE SÃo Paulo

BRAZIL

E-mail address: tausk@ime.usp.br 